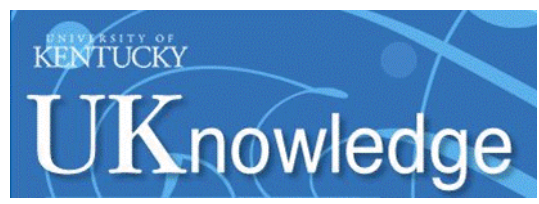

University of Kentucky

UKnowledge

$1-2009$

\title{
The Three-Dimensional Dynamic Structure of the Inner Orion Nebula
}

C. R. O'Dell

Vanderbilt University

W. J. Henney

Universidad Nacional Autónoma de México, Mexico

N. P. Abel

University of Cincinnati

Gary J. Ferland

University of Kentucky, gary@uky.edu

S. J. Arthur

Universidad Nacional Autónoma de México, Mexico

Follow this and additional works at: https://uknowledge.uky.edu/physastron_facpub

Part of the Astrophysics and Astronomy Commons, and the Physics Commons

Right click to open a feedback form in a new tab to let us know how this document benefits you.

\section{Repository Citation}

O'Dell, C. R.; Henney, W. J.; Abel, N. P.; Ferland, Gary J.; and Arthur, S. J., "The Three-Dimensional Dynamic Structure of the Inner Orion Nebula" (2009). Physics and Astronomy Faculty Publications. 132.

https://uknowledge.uky.edu/physastron_facpub/132

This Article is brought to you for free and open access by the Physics and Astronomy at UKnowledge. It has been accepted for inclusion in Physics and Astronomy Faculty Publications by an authorized administrator of UKnowledge. For more information, please contact UKnowledge@lsv.uky.edu. 


\section{The Three-Dimensional Dynamic Structure of the Inner Orion Nebula}

\section{Digital Object Identifier (DOI)}

http://dx.doi.org/10.1088/0004-6256/137/1/367

\section{Notes/Citation Information}

Published in The Astronomical Journal, v. 137, no. 1, p. 367-382.

(C) 2009. The American Astronomical Society. All rights reserved.

The copyright holder has granted permission for posting the article here. 


\title{
THE THREE-DIMENSIONAL DYNAMIC STRUCTURE OF THE INNER ORION NEBULA*
}

\author{
C. R. O’'Dell ${ }^{1}$, W. J. Henney ${ }^{2}$, N. P. Abel ${ }^{3}$, G. J. Ferland ${ }^{4}$, and S. J. Arthur ${ }^{2}$ \\ ${ }^{1}$ Department of Physics and Astronomy, Vanderbilt University, Box 1807-B, Nashville, TN 37235, USA; cr.odell@vanderbilt.edu \\ ${ }^{2}$ Centro de Radioastronomía y Astrofísica, Universidad Nacional Autónoma de México, Apartado Postal 3-72, 58090 Morelia, Michaoacán, Mexico \\ ${ }^{3}$ Department of Mathematics and Physics, College of Applied Science, University of Cincinnati, Cincinnati, OH 45221, USA \\ ${ }^{4}$ Department of Physics and Astronomy, University of Kentucky, Lexington, KY 40506, USA \\ Received 2008 August 14; accepted 2008 October 22; published 2008 December 15
}

\begin{abstract}
The three-dimensional structure of the brightest part of the Orion Nebula is assessed in the light of published and newly established data. We find that the widely accepted model of a concave blister of ionized material needs to be altered in the southwest direction from the Trapezium, where we find that the Orion-S feature is a separate cloud of very optically thick molecules within the body of ionized gas, which is probably the location of the multiple embedded sources that produce the optical and molecular outflows that define the Orion-S star formation region. Evidence for this cloud comes from the presence of $\mathrm{H}_{2} \mathrm{CO}$ lines in absorption in the radio continuum and discrepancies in the extinction derived from radio-optical and optical-only emission. We present an equilibrium Cloudy model of the Orion-S Cloud, which successfully reproduces many observed properties of this feature, including the presence of gas-phase $\mathrm{H}_{2} \mathrm{CO}$ in absorption. We also report the discovery of an open-sided shell of [O III] surrounding the Trapezium stars, revealed through emission-line ratio images and the onset of radiation shadows beyond some proplyds. We show that the observed properties of the shell are consistent with it being a stationary structure, produced by shock interactions between the ambient nebular gas and the high-velocity wind from $\theta^{1}$ Ori C. We examine the implications of the recently published evidence for a large blueshifted velocity of $\theta^{1}$ Ori $\mathrm{C}$ with respect to the Orion molecular cloud, which could mean that this star has only recently begun to photoionize the Orion Nebula. We show that current observations of the nebula do not rule out such a possibility, so long as the ionization front has propagated into a pre-existing low-density region. In addition, a young age for the nebula would help explain the presence of nearby proplyds with a short mass-loss timescale to photoablation.
\end{abstract}

Key words: H II regions - ISM: individual (Orion Nebula, NGC 1976) - stars: formation

\section{INTRODUCTION}

The brightest portion of NGC 1976, the Orion Nebula, is commonly called the Huygens region (after Christiaan Huygens, who published the first drawing of the nebula in 1659) and its form in three dimensions was the subject of many early papers (reviewed in O'Dell 2001a). The presently accepted basic model of a photoionized thin layer of gas flowing off the side of the Orion molecular cloud (OMC) facing us was invoked to explain the progressive blueshift of the emission lines with respect to the OMC (Zuckerman 1973; Ballick et al. 1974). The bright bar along the southeast border of the Huygens region is a location where the main ionization front (MIF) is tilted almost along the line of sight to the observer. The other portions of the MIF have been mapped in three dimensions by Wen \& O'Dell (1995) by a method that assumes all the ionizing radiation arises from the $\mathrm{O} 7 \mathrm{Vp} \operatorname{star} \theta^{1} \mathrm{Ori} \mathrm{C}$, and then calculates the distance to the MIF that would satisfy the known conditions of gas density and observed $\mathrm{H} \alpha$ surface brightness, this being a broader application of a method first presented in Baldwin et al. (1991). Although this three-dimensional (3D) map has the limitation that it becomes progressively less accurate as one moves away from the line of sight toward $\theta^{1}$ Ori $\mathrm{C}$, it did establish that $\theta^{1}$ Ori $\mathrm{C}$ lies about ${ }^{5} 0.2 \mathrm{pc}$ in front of the MIF,

\footnotetext{
* Based on observations with the NASA/ESA Hubble Space Telescope, obtained at the Space Telescope Science Institute, which is operated by the Association of Universities for Research in Astronomy, Inc., under NASA Contract No. NAS 5-26555.

5 Throughout this paper we will adopt a distance of $440 \mathrm{pc}$ to the Orion Nebula, a value derived (O'Dell \& Henney 2008) using the results of what are currently thought to be the best independent determinations. Earlier papers that used different assumed distances will have their results scaled to this distance.
}

confirmed the structure in the bright bar region, and showed that the nebula was otherwise a concave surface with a large bump to the southwest of $\theta^{1}$ Ori $\mathrm{C}$ in the Orion-S star formation region. The surface brightness near $\theta^{1}$ Ori $\mathrm{C}$ can be explained as emission by a constant density layer of about $0.1 \mathrm{pc}$ thickness (Pogge et al. 1992). This is only a reference number since the emissivity must be much higher near the MIF and drops as the square of the density. In a nebula where there is free expansion of the photoionized gas and there is a single dominant ionizing star, a concave shape of the MIF is the natural result, with bumps, valleys, and ridges reflecting underlying conditions of the host molecular cloud (MC). The ionization front is expected to be closer to the ionizing star where the MC density is higher and further away where the underlying density is lower. The ionized gas density drops rapidly away from the MIF, but it is not clear what the density is in the immediate vicinity of $\theta^{1}$ Ori $\mathrm{C}$ since it is expected that its intense high-velocity wind would create a hot, low-density cavity around it. This cavity has not been detected directly except for observations of stand-off shocks in front of the proplyds closest to $\theta^{1}$ Ori C (Bally et al. 1998, 2000).

In addition to the rich Orion Nebula Cluster (ONC) centered on the bright Trapezium stars, there are two star formation centers imbedded in the OMC. The first is associated with the deeply imbedded (0.2 pc; Doi et al. 2004) BN-KL infrared (IR) and radio sources to the northwest of $\theta^{1}$ Ori $\mathrm{C}$ and the second is in the Orion-S region.

With the discovery of $21 \mathrm{~cm}$ absorption lines in the radio continuum spectrum (van der Werf \& Goss 1989) it was recognized that there was a Veil of neutral material on the observer's side of the nebula, and subsequent absorption line spectroscopy (Abel et al. 2004b, 2006) established its physical 
characteristics and approximate location of about 1 parsec on the observer's side (henceforth foreground) of $\theta^{1}$ Ori C. Recent detailed reviews have covered the ONC (Muench et al. 2008) and the nebula plus obscured star formation regions (O'Dell et al. 2008, and see also O'Dell 2001a, 2001b).

The Huygens region occupies the northeast corner of a much larger structure called the extended Orion Nebula (EON; Güdel et al. 2008). It is known that there is a systematic flow of ionized material into the EON (O'Dell 2001a; Henney et al. 2005), but the lower surface brightness has limited the number of investigations of this region (Subrahmanyan et al. 2001). However, the EON is the location of two X-ray bright regions of hot plasma (Güdel et al. 2008). High-resolution optical (Henney et al. 2007) and IR (Megeath \& Robberto 2006) images of the EON are now available. Although these images show many interesting large-scale features, there is neither the detail nor abundance of stars of the Huygens region.

In this paper, we will present the most relevant information about the ONC and the Orion Nebula, then integrate this into a modified picture of the Orion Nebula's 3D structure and its history. In Section 2 we present the most useful information, then in Section 3 give the results of where this information leads.

\section{BACKGROUND INFORMATION}

\subsection{Emission-Line Images of the Orion Nebula}

The Huygens region has been the subject of numerous imaging studies. Arguably the most useful ground-based study is that of Pogge et al. (1992), which utilized a Fabry-Perot system to isolate emission from the $\mathrm{H} \alpha, \mathrm{H} \beta$, [O III] $5007 \AA$, [N II] $6583 \AA$ and $6548 \AA$, [S II] $6716 \AA$ and $6731 \AA$, and [He I] $6678 \AA$ lines. O'Dell \& Wong (1996) presented a mosaic of the Hubble Space Telescope (HST) Wide Field Planetary Camera 2 (WFPC2) images at the superior resolution of the HST. The particular advantage of the HST images is that the WFPC2 images allow clear discrimination of not only the isolated [O III] $5007 \AA$ line, but also both the $\mathrm{H} \alpha 6563 \AA$ line and the nearby [N II] $6583 \AA$ line in addition to the filters being narrow enough that they provide a good isolation of the emission lines against the strong scattered light continuum that primarily arises from dust grains in the dense region immediately behind the MIF (O’Dell \& Doi 1999). A later HST survey (Henney et al. 2007) with the Advanced Camera for Surveys (ACS) covered a wider field of view with pixels one half the angular size of those in the WFPC2, but the filters used do not allow a clear delineation of the important emission lines (O'Dell 2004). The Huygens region has been mapped with the VLA at about 1".7 resolution (O'Dell \& Yusef-Zadeh 2000) at the extinction free $20.5 \mathrm{~cm}$ continuum. By comparing the surface brightness calibrated (O'Dell \& Doi 1999) images and the radio images, it was possible to derive a map of the optical extinction across the Huygens region and to generate extinction-corrected versions of the HST WFPC2 emission-line images (O’Dell \& Yusef-Zadeh 2000). In this study, we employ the several forms of the WFPC2 images, all processed in part using the IRAF package. ${ }^{6}$ We see in Figure 1 that the Orion-S region to the southwest of the Trapezium resembles the bright bar region in being enhanced in lowionization $\left[\mathrm{N}_{\mathrm{II}}\right]$ emission and being much brighter than adjacent

\footnotetext{
6 IRAF is distributed by the National Optical Astronomy Observatories, which is operated by the Association of Universities for Research in Astronomy, Inc., under cooperative agreement with the National Science Foundation.
}

areas. These characteristics are consistent with the brightest part of the central Huygens region being both closer to $\theta^{1}$ Ori $\mathrm{C}$ and also being an inclined face of ionized gas. This picture is consistent with the results of the 3D modeling of Wen \& O'Dell (1995).

\subsection{The Orion Nebula's Veil}

Although the optical extinction of the Veil has been recognized for some time, it has been established only recently (O'Dell et al. 1992; O'Dell 2002) that most of this extinction arises from the near side of the ionized zone and not within it. The detailed analysis of Abel et al. $(2005,2006)$ established that the physical conditions in the two $21 \mathrm{~cm} \mathrm{H}$ I absorption velocity components of the Veil are rather different, the energy density in their component A being dominated by the magnetic field measured from the Zeeman effect. The elements C, S, Mg, and Si are ionized in the Veil by the far-ultraviolet (FUV; 5-13.6 eV) radiation that penetrates it, even though the Veil is optically thick to extreme-ultraviolet (EUV) radiation. There must be a secondary hydrogen ionization front associated with the Veil and on the far side (away from the observer and closer to $\theta^{1}$ Ori $C$ ) of the Veil. Abel et al. (2006) identify emission lines probably arising from the Veil's ionization front.

The radial velocities of the various emission and absorption line components along the line of sight through the Trapezium are summarized in Table 1.

\section{3. $\mathrm{H}_{2} \mathrm{CO}$ is Seen in Absorption in Orion-S}

An early study (Johnston et al. 1983) at $16^{\prime \prime}$ resolution detected $\mathrm{H}_{2} \mathrm{CO}$ in absorption against the radio continuum with the location identified as Orion-S, a region with multiple known molecular emission lines (O'Dell et al. 2008) and well-defined bipolar molecular outflows in $\mathrm{CO}$ (Zapata et al. 2005) and $\mathrm{SiO}$ (Zapata et al. 2006). A higher resolution $\left(5^{\prime \prime} .1 \times 7^{\prime \prime} .6\right)$ study (Mangum et al. 1993) confirmed the presence of this absorption feature and the results are shown in Figure 1 and Table 1. In Figure 1 we see that the $\mathrm{H}_{2} \mathrm{CO}$ absorption is distinct from the bipolar outflows, the strongest IR and radio sources, and the sources of the high-velocity optical outflows (O'Dell \& Henney 2008). Mangum et al. (1993) point out that the presence of $\mathrm{H}_{2} \mathrm{CO}$ in absorption means that the cloud containing it must lie in front of the ionized gas, a position also taken in more general form by Johnston et al. (1983) and Wilson et al. (2001). Since $\mathrm{H}_{2} \mathrm{CO}$ can only exist in a cold dense gas that is optically thick to FUV (and therefore also EUV) radiation, there will be a corresponding high extinction at visual wavelengths. Therefore, we have looked for optical extinction associated with this feature with the results described in the next section.

\subsection{Anomalies in the Extinction in the Orion-S Region}

The optical appearance of the Huygens region is strongly affected by extinction occurring within the Veil. The clearest example is the Dark Bay feature to the east-northeast of the Trapezium. This extinction generally decreases away from the Dark Bay as the line of sight is moved to the southwest. The extinction has been derived in several fashions. In slit spectroscopy, the common approach has been to compare the flux ratios of the strongest Balmer series lines with the ratios expected from theoretical predictions calculated for the local conditions (primarily the electron temperature). A good example of this is the study of Baldwin et al. (1991), who obtained sample spectra along a well-defined east-west path beginning 


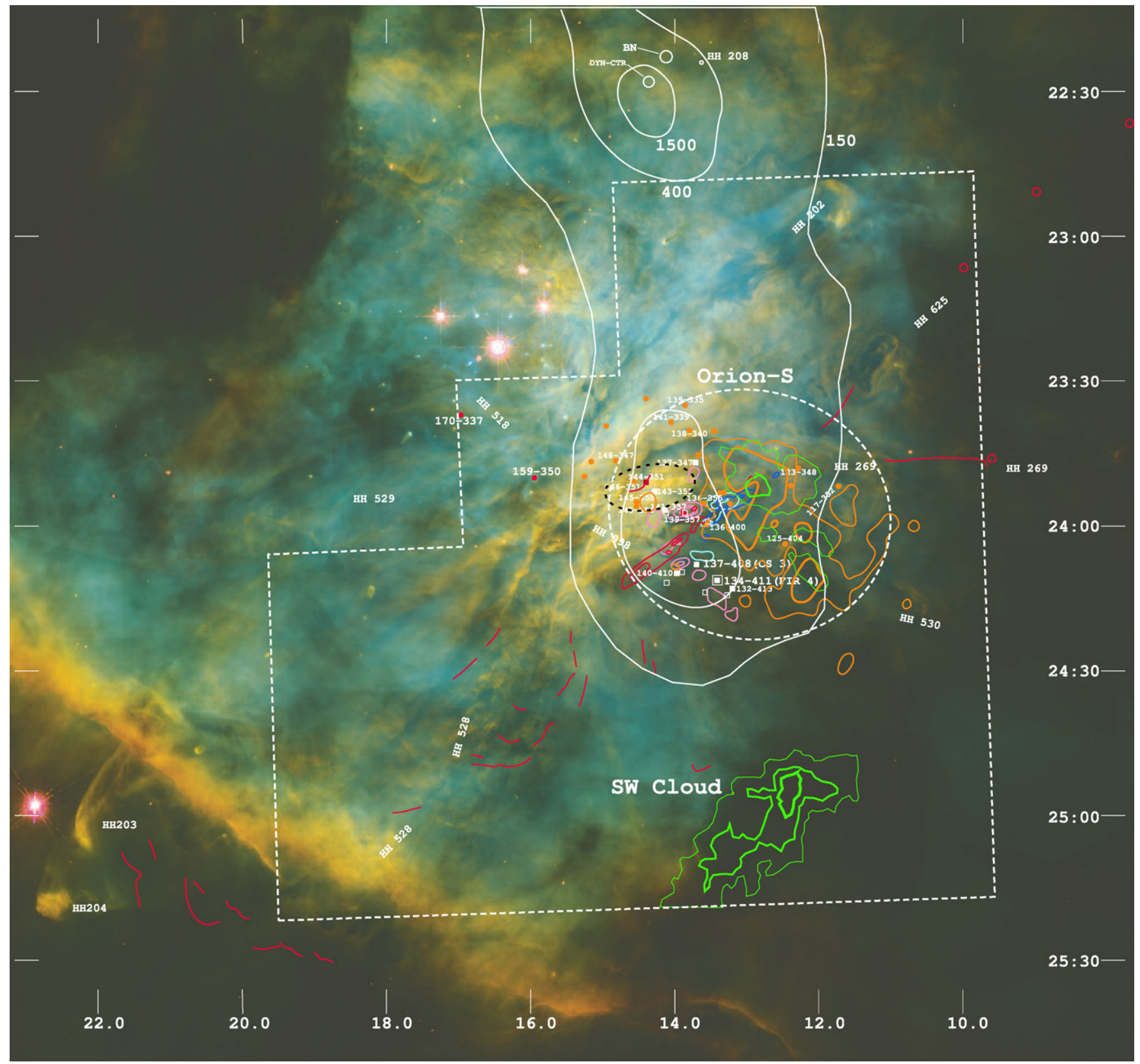

Figure 1. This $233^{\prime \prime} \times 219^{\prime \prime}$ image is composed of a mosaic of WFPC2 images (O’Dell \& Wong 1996) with F502N ([O III]) as blue, F656N (H $\left.\alpha\right)$ as green, and F658N ([N II]) as red. North is up and the labels along the edge depict the right ascension beyond 5:30:00 and the declination south of $-5: 20: 00$ (2000). Major outflow features are labeled in addition to objects discussed in the text. Throughout this paper a position-based designation is used (O'Dell \& Wen 1994) except for large individual features such as Herbig Haro objects. The white dashed ellipse encloses the smaller features collectively discussed in the text as the Orion-S feature. The red lines and circles represent strong IR $\mathrm{H}_{2}$ features (Kaify et al. 2000; Stanke et al. 2002) not associated with the BN-KL deeply embedded sources. The strong dark red/blue contoured lines indicate $\mathrm{CO}$ outflow (Zapata et al. 2005) and the pastel-colored pink/light-blue contoured lines indicate SiO outflow (Zapata et al. 2006). The orange contours are $\mathrm{H}_{2} \mathrm{CO}$ absorption features (Mangum et al. 1993) at the level of $-80 \mathrm{~K} \mathrm{~km} \mathrm{~s}^{-1}$ (heavier) and $-50 \mathrm{~K} \mathrm{~km} \mathrm{~s}^{-1}$ (lighter). The green contours of increasing thickness depict regions of the difference of extinction $\left(c_{\mathrm{H} \beta}^{\mathrm{DIF}}\right.$ as defined in Section 2.4) of 0.1, 0.2, and 0.3. The sharp boundary of the more southerly $c_{\mathrm{H} \beta}^{\mathrm{DIF}}$ excess feature is the result of reaching the edge of the WFPC2 field of view. The region most likely to contain the sources of the high-velocity optical outflows is shown as a dark dashed ellipse (O'Dell \& Henney 2008). The open squares indicate the positions of $\mathrm{H}_{2} \mathrm{O}$ maser sources (Gaume et al. 1998). The point sources within the dashed outline are coded by the shortest wavelength of their detection, with filled white squares indicating the positions of radio-only visible sources (Zapata et al. 2004a, 2004b, 2005), red squares the positions of sources seen only in the mid-IR (Smith et al. 2004; Robberto et al. 2005), and filled orange circles the positions of stars in the near-IR catalog of Hillenbrand \& Carpenter (2000). The white contours show the $350 \mu \mathrm{m}$ emission in this area (Houde et al. 2004) in units of Jy per 12" beam. The irregular dashed white line indicates the field where $c_{\mathrm{H} \beta}$ was determined both by the radio/optical and optical line ratio methods.

about $30^{\prime \prime}$ west of $\theta^{1}$ Ori $\mathrm{C}$ and derived the extinction by comparing Paschen lines with $\mathrm{H} \gamma$. The highest spatial resolution study is that of O'Dell et al. (2003), where calibrated HST WFPC2 $\mathrm{H} \alpha$ and $\mathrm{H} \beta$ images from a single WFPC2 pointing to the southwest of the Trapezium were compared with theory. Pixel-by-pixel extinction corrections were obtained as part of a study of electron-temperature fluctuations. The widest field-ofview high-resolution determination of the extinction was that of O'Dell \& Yusef-Zadeh (2000) who compared the H $\alpha$ surface brightness of a mosaic of Gaussian blurred HST WFPC2 images with VLA $20 \mathrm{~cm}$ images obtained at $1^{\prime \prime} .7$ resolution. In all these studies similar extinction curves were used (Costero \& Peimbert 
Table 1

Heliocentric Velocities of the Major Orion Nebula Components

\begin{tabular}{|c|c|c|c|}
\hline Designation & Components & $V_{\odot}\left(\mathrm{km} \mathrm{s}^{-1}\right)$ & Source \\
\hline$V_{\mathrm{OMC}}$ & Molecules & $25.8 \pm 1.7$ & O’Dell et al. (2008) \\
\hline$V_{\mathrm{MIF}}$ & {$[\mathrm{O} I],[\mathrm{S} \mathrm{II}]$} & $25.5 \pm 1$ & O’Dell (2001b) \\
\hline$V_{\mathrm{MIL}}$ & [O II], [O III], [N II $],\left[\mathrm{Cl}_{\text {III }}\right], \mathrm{H}^{+}, \mathrm{He}^{+}$ & $18.0 \pm 1.4$ & O’Dell (2001b) \\
\hline$V_{\text {Trap }}$ & $\theta^{1}$ Ori $\mathrm{A}, \theta^{1}$ Ori $\mathrm{B}, \theta^{1}$ Ori $\mathrm{D}$ & $24 \pm 3$ & Abt et al. (1991) \\
\hline$V_{\text {Cluster }}$ & Lower mass stars & $25.8 \pm 1.0$ & Fürész et al. (2008) \\
\hline$V_{\theta^{1} \text { OriC }}$ & $\theta^{1}$ Ori C & 13 & Stahl et al. (2008) \\
\hline$V_{\text {Arc }}$ & Big Arc & $9.2 \pm 1$ & This study \\
\hline$V_{\text {Veil- }} \mathrm{H}^{+}$ & $\mathrm{S}^{++}, \mathrm{P}^{++},[\mathrm{N}$ II $], \mathrm{He}$ I, [O II] & $3 \pm 2$ & Abel et al. (2006) \\
\hline$V_{\mathrm{HI}-\mathrm{A}}$ & H I & $23.4 \pm 0.01$ & Abel et al. (2006) \\
\hline$V_{\mathrm{HI}-\mathrm{B}}$ & Н I & $19.3 \pm 0.03$ & Abel et al. (2006) \\
\hline$V_{\mathrm{H}_{2} \mathrm{CO}}$ & $\mathrm{H}_{2} \mathrm{CO}$ absorption & $28.6 \pm 1.6$ & Mangum et al. (1993) \\
\hline
\end{tabular}

Note. Local standard of rest velocities can be obtained by subtracting $18.1 \mathrm{~km} \mathrm{~s}^{-1}$ from $V_{\odot}$ values.

1970; Cardelli et al. 1989). The derived extinction is commonly expressed as $c_{\mathrm{H} \beta}$, the logarithm of the extinction at $4861 \AA$, the wavelength of $\mathrm{H} \beta$. In this work, we will refer to the $c_{\mathrm{H} \beta}$ value derived from the comparison of the radio continuum and $\mathrm{H} \alpha$ as $c_{\mathrm{H} \beta}^{\mathrm{VLA}}$ and to the value derived from comparison of WFPC2 Balmer $\mathrm{H} \alpha$ and $\mathrm{H} \beta$ images as $c_{\mathrm{H} \beta}^{\mathrm{H} \alpha}$, while the difference of the two will be $c_{\mathrm{H} \beta}^{\mathrm{DFF}}=c_{\mathrm{H} \beta}^{\mathrm{VLA}}-c_{\mathrm{H} \beta}^{\mathrm{H} \alpha}$.

Although a comparison of the results for $c_{\mathrm{H} \beta}^{\mathrm{VLA}}$ and $c_{\mathrm{H} \beta}^{\mathrm{H} \alpha}$ for nine well-sampled smaller regions showed that there was a good agreement of the results of the two methods for finding $c_{\mathrm{H} \beta}$ (ratio of $0.93 \pm 0.19$; O'Dell \& Yusef-Zadeh 2000), there is reason to expect that the two methods should not agree exactly.

The first reason would be the effect of scattering of the optical lines by dust in the dense photon-dominated region (PDR) that lies immediately beyond the MIF. This scattering is what produces a broad redshifted component in the high-resolution spectra of the intrinsically narrower heavy ion emission lines and it must also be present in the Balmer lines. This is why the continuum of the Orion Nebula is much stronger than expected from simply atomic processes (Baldwin et al. 1991), in effect, the Orion Nebula is also a bright reflection nebula. One would expect the effect of this process to cause an underestimate of $c_{\mathrm{H} \beta}^{\mathrm{VA}}$ and would affect $c_{\mathrm{H} \beta}^{\mathrm{H} \alpha}$ less since one is dealing with only the difference in the amount of scattering of the $\mathrm{H} \alpha$ and $\mathrm{H} \beta$ lines, rather than the absolute amount at $\mathrm{H} \alpha$, as done in deriving $c_{\mathrm{H} \beta}^{\mathrm{VLA}}$. Taking the fraction of the light scattered in the forbidden lines, this position-dependent effect should only be a few hundredths in the logarithm (dex).

The second reason to expect $c_{\mathrm{H} \beta}^{\mathrm{DIF}}$ to be nonzero is that when using only the optical emission (the Balmer line ratio method) one is dealing entirely with radiation that has not suffered a large amount of extinction, whereas the radio continuum to $\mathrm{H} \alpha$ method can be thrown off by the fact that some of the total radio continuum is produced by volumes behind regions that are very optically thick to $\mathrm{H} \alpha$. In this case, one would be using a radio signal from all along the line of sight but $\mathrm{H} \alpha$ emission from only in front of the obscuring material and $c_{\mathrm{H} \beta}^{\mathrm{VLA}}$ would be too large, i.e. $c_{\mathrm{H} \beta}^{\mathrm{DIF}}$ would be positive.

The third reason for a difference would be variations in the ratio of total-to-selective extinction. Derivation of $c_{\mathrm{H} \beta}^{\mathrm{H} \alpha}$ depends upon the reddening curve adopted, which is primarily determined by the ratio of total-to-selective extinction, while $c_{\mathrm{H} \beta}^{\mathrm{VLA}}$ is less dependent on this ratio.

Since the presence of $\mathrm{H}_{2} \mathrm{CO}$ in absorption indicates that near Orion-S there is a region where radio continuum emission occurs in part behind the totally obscuring cloud, we looked for significant variations in $c_{\mathrm{H} \beta}^{\mathrm{DIF}}$ in the region southwest of the Trapezium where this could be done. The results are also shown in Figure 1, where we have superimposed the contours of large values of $c_{\mathrm{H} \beta}^{\mathrm{DIF}}$. One sees that there are two regions where $c_{\mathrm{H} \beta}^{\mathrm{DIF}}$ is large, the northerly one nearly coinciding with the $\mathrm{H}_{2} \mathrm{CO}$ absorption and the other coinciding with the distinct obscuring feature to its south designated as the stellar wind (SW) cloud (O'Dell \& Yusef-Zadeh 2000). This means that in both these regions optical emission lines are recording only part of the ionized gas along the lines of sight. Another way of wording this is that in these regions the model of the MIF based on a single dominant emitting layer cannot be accurate. Since the Wen \& O'Dell (1995) study used the reddening-corrected $\mathrm{H} \alpha$ line for reference, in these regions the model will be a depiction of the closer (to the observer) emitting region. This means that the "bump" in the Orion-S region is there, but it is not simply the result of a nearer part of the MIF, but it is an isolated optically thick feature directly ionized on its surface by $\theta^{1}$ Ori $\mathrm{C}$ and behind it is another ionized region. The more southerly region of large $c_{\mathrm{H} \beta}^{\mathrm{DIF}}$ is probably similar, but it must not be as dense as the Orion-S feature since one does not see $\mathrm{H}_{2} \mathrm{CO}$ in absorption.

\subsection{Radiation Shadows in the Orion Nebula}

Nature provides a way of probing where material is located within the Orion Nebula through the ionization shadows created beyond the proplyds. In a study of linear features within the Orion Nebula and the Helix Nebula, O'Dell (2000) established that these features can occur when there is a single dominant ionizing star and an intervening object optically thick to the EUV Lyman continuum (LyC) radiation. The first-order theory for this situation had already been determined (Cantó et al. 1998). Gaseous material lying beyond an object like a proplyd (in the Orion Nebula) or a knot (in the Helix Nebula) will be shadowed from photoionizing LyC photons coming directly from the ionizing star, but will be exposed to LyC photons produced through the recombination of surrounding nebular gas. This diffuse radiation field will be a small fraction of the direct radiation from the ionizing star and there will be fewer higher energy photons than in the direct radiation. The difference in the radiation field is because the recombinations occur preferentially to energy states just above hydrogen's ionization threshold. The lower density of ionizing photons means a lower rate of photoionization and the different energy distribution means that any shadowed gas will equilibrate at a lower electron temperature. The lower temperature means 


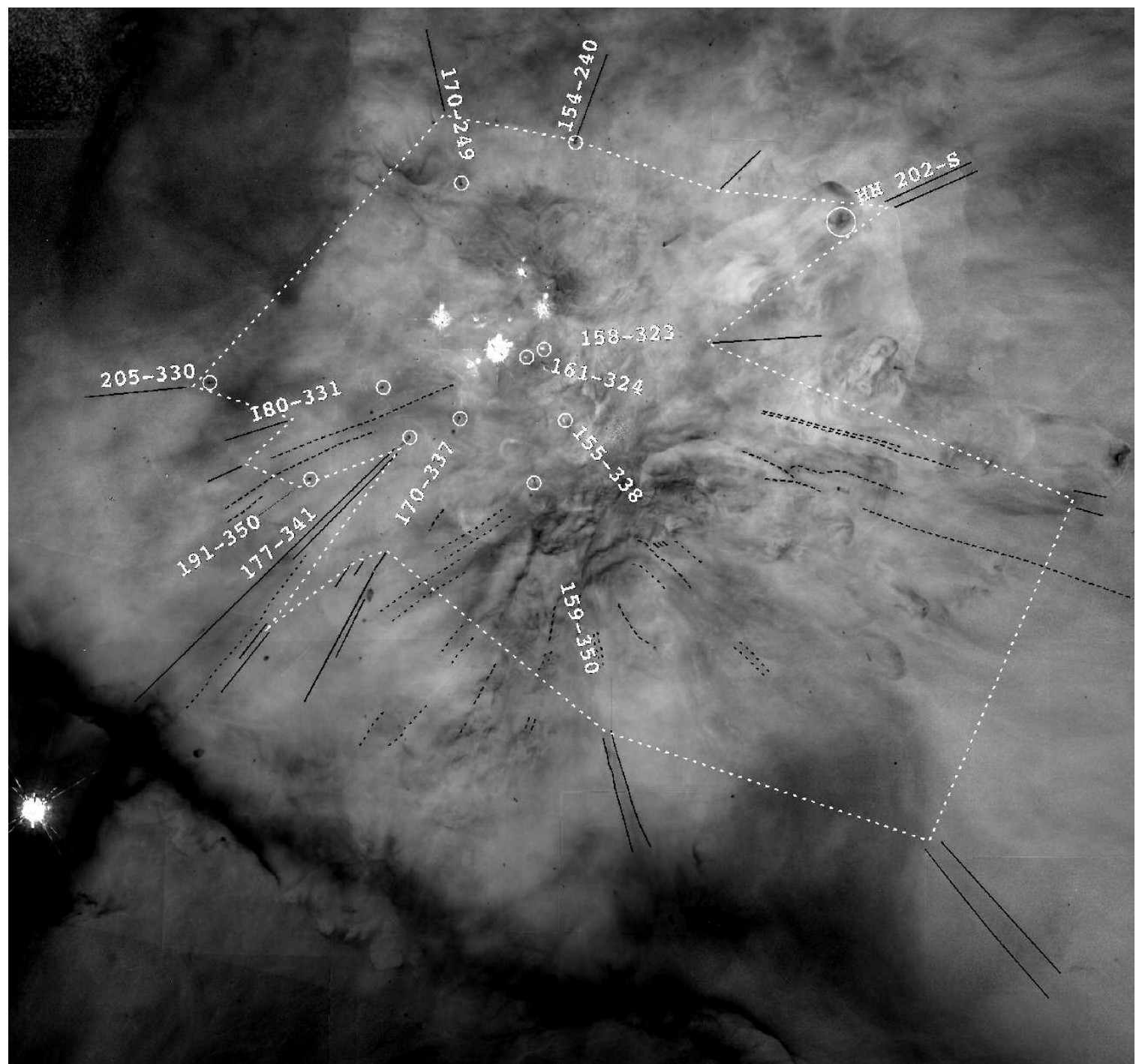

Figure 2. Same field of view as in Figure 1 is shown except that here the ratio of the F502N over F656N images is shown. The solid lines depict radial ionization shadow features that project back toward $\theta^{1}$ Ori $\mathrm{C}$ and the dotted lines depict significant, nearly linear features that do not project back to $\theta^{1}$ Ori $\mathrm{C}$. Almost all of the solid-line features have an identified proplyd, which is the occulting source and in this case the designation of the proplyd is given, adjacent to the proplyd's position. The exception to this rule is the ionization shadow feature lying beyond the HH 202-S shock. The white dashed line shows the boundary where radial shadows begin.

that forbidden line radiation will have a reduced emissivity and recombination lines will have an increased emissivity. This shows up particularly well when looking at the ratio of images in [O III] and $\mathrm{H} \alpha$, as shown in Figures 2 and 3. O'Dell (2000) established that the linear rays seen in the $\left[\mathrm{O}_{\mathrm{III}}\right] / \mathrm{H} \alpha$ image occurred along lines of sight passing through $\theta^{1}$ Ori $\mathrm{C}$ and a proplyd, but not all proplyds have visible radiation shadows.

These shadowed regions will only appear when there is material present. This means that the absence of a radiation shadow indicates that only low-density gas lies within that conic column. In the case where the radiation shadow does not begin at the proplyd, then the gas occupying the space between the proplyd and the start of the radiation shadow must be of low density.

Figure 2 has had all the linear and nearly linear features traced, solid lines being used for the indistinguishably linear features and dashed lines for the nearly linear features (which cannot be radiation shadows unless second-order effects discussed in O'Dell 2000 are in play). One sees that in essentially every case of a linear feature there is an identified shadowing proplyd and, again, that not all proplyds have radiation shadows. In many cases, the associated proplyd is well inside the radiation shadow (closer to $\theta^{1}$ Ori $\mathrm{C}$ ). The general pattern is clear, none of the proplyds near $\theta^{1}$ Ori $C$ have radiation shadows extending close to them and the proplyds with closely approaching radiation shadows are distant from $\theta^{1}$ Ori $\mathrm{C}$. We interpret this to mean that there is a region of low-density (or very different ambient condition) gas lying within the approximate boundary shown in Figure 2. This irregular boundary in the plane of the sky is the projection along the line of sight and the central cavity is probably somewhat larger than the outlined boundary. The irregular boundary drawn indicates that there is an asymmetric distribution about the dominant ionizing star $\theta^{1}$ Ori C.

It should be noted that the high-velocity feature HH 202-S (O'Dell \& Henney 2008) also casts a radiation shadow. This indicates that this shock produces a sufficiently high-density gas that it is optically thick to LyC photons and becomes a local ionization front within a generally ionized volume. This vitiates any argument that $\mathrm{HH} 202$ was caused by high-velocity material from the Orion-S region impinging on either the foreground Veil or a section of the MIF that had curved toward the observer and intercepted the collimated outflow. 


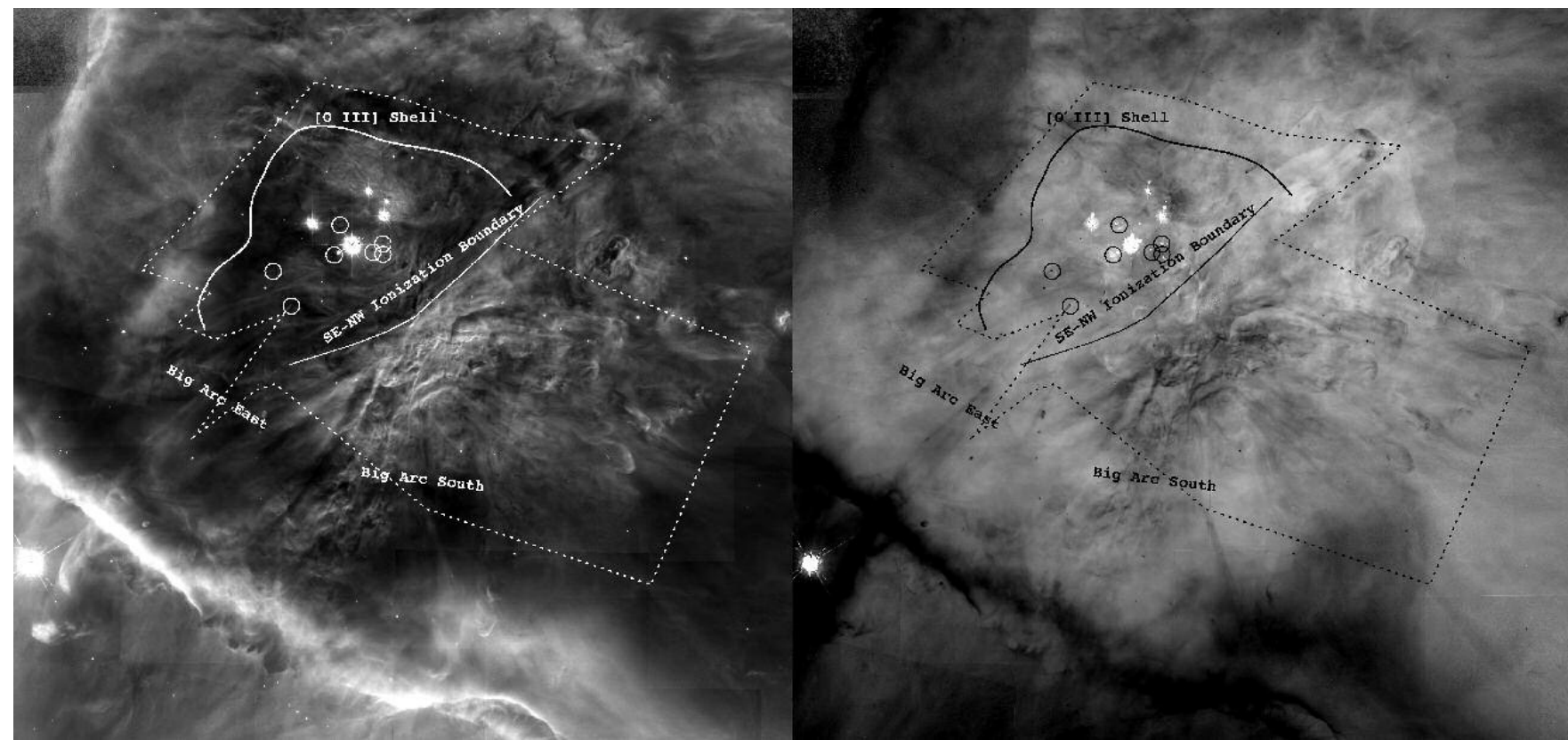

Figure 3. Same field of view as in Figures 1 and 2 is shown in both panels. The left panel shows the ratio of F658N/F656N ([N II]/H $\alpha$ ) and the right panel shows the ratio of $\mathrm{F} 502 \mathrm{~N} / \mathrm{F} 656 \mathrm{~N}([\mathrm{O} \mathrm{III}] / \mathrm{H} \alpha)$. Both are intended to show the systematic ionization changes across the Huygens region. The left panel is little affected by local variations in interstellar reddening, while the right panel is. The dashed line indicates the boundary where radial shadows begin. The inner boundary of the [O III] shell feature discussed in Section 2.6 is outlined with a curved solid line. The lighter solid line indicates the southwest boundary of a high-ionization region close to the Trapezium stars. The circles are centered on the proplyds that have stand-off shocks, which indicate that they lie within the unshocked hypersonic SW from $\theta^{1}$ Ori $\mathrm{C}$.

\subsection{Large-Scale Ionization Structures within the inner Huygens Region}

Within the paradigm of the Orion Nebula being described to first order as a relatively thin blister of ionized gas on the $\theta^{1}$ Ori $\mathrm{C}$ side of the MIF, one expects that the [NII] emitting zone will be quite narrow as compared with the $\mathrm{H} \alpha$ emitting zone, which includes all of the ionized gas. [O $\mathrm{OII}]$ emission must arise from closer to $\theta^{1}$ Ori $\mathrm{C}$ and over a larger volume since the effective temperature of $\theta^{1}$ Ori $\mathrm{C}$ is insufficient to produce a $\mathrm{He}^{++}$zone (where $\mathrm{O}^{++}$has been ionized and [O III] is not emitted). This means that the $[\mathrm{N}$ III $/ \mathrm{H} \alpha$ ratio is a good indicator of when the MIF is highly tilted with respect to the plane of the sky. This is the reason why the bright bar feature is so well delineated in the left panel of Figure 3. Although there are many unexplained features of the bright bar, the overwhelming evidence is that it is an escarpment in the MIF where we see almost along the local ionization front. This is probably also the correct interpretation of the low-ionization zone to the southwest of the Trapezium that lies on the northeast side of Orion-S.

\subsubsection{A Previously Unrecognized High-Ionization Structure within the Inner Huygens Region}

Figure 3 (left panel) reveals an unexpected large-scale structure in a $\mathrm{C}$-shaped zone of low $\left[\mathrm{N}_{\mathrm{II}}\right] / \mathrm{H} \alpha$ with $\theta^{1}$ Ori C located near its middle. Close examination of Figure 3 (right panel) indicates that where $[\mathrm{NII}]$ emission is weak, $\left[\mathrm{O}_{\mathrm{III}}\right]$ emission is strong. This means that the $\mathrm{C}$-shaped zone is an ionization phenomenon. The fact that the [N $\mathrm{NI}]$ deficit and [O III] excess is an irregular line, rather than a closed area, indicates that this is a shell of high ionization. Henceforth we will call this the [O III] shell, with full recognition that this feature is probably not closed and we do not understand its 3D characteristics. South of the Trapezium and Orion-S lies a large high-ionization feature labeled as Big Arc South and Big Arc East. This was originally discovered in a low-velocity resolution study (O'Dell et al. 1997), then included in a later higher resolution study (Doi et al. 2004), and the correct interpretation as a partial shell blueshifted about $V_{\odot}=9 \mathrm{~km} \mathrm{~s}^{-1}$ being presented in García-Díaz et al. (2008) and confirmed by our remeasuring a sample of the Big Arc from the [O III] spectra data set (Doi et al. 2004). Although the Big Arc is seen in projection to be almost continuous with the southern arm of the [O III] shell, it is very distinct kinematically, and the physical relation between these two features remains unclear.

The approximate alignment of the boundaries of the radiation shadows and the [O III] shell argues that this is an incomplete shell with lower central density within the larger mass of ionized gas. It is not an ionization boundary because one does not see [N II] enhancement on its outer perimeter. The feature seems to open to the west-southwest, being most open in the direction of Orion-S. There is a nearly linear border to the high-ionization zone, which we have labeled in Figure 3 as the southeastnorthwest ionization boundary. It is not immediately obvious that this feature is an ionization boundary or caused by the higher extinction of the foreground Orion-S Cloud (see Section 3.1).

Figure 3 also shows the boundary of the radiation shadows from Figure 2 and we see that there is good agreement of this boundary with the $[\mathrm{O}$ III] shell, which further strengthens the argument that this [O $\mathrm{III}]$ excess traces the outer boundary of a volume of low-density gas. We also show in Figure 3 the location of proplyds with stand-off shocks and find that all of them fall within the $\mathrm{C}$-shaped [O III] shell. Since it is most likely that these stand-off shocks are formed by direct exposure to the high-velocity $\mathrm{SW}$ of $\theta^{1}$ Ori $\mathrm{C}$, this argues that the [O $\left.\mathrm{III}\right]$ shell is created by that SW, an idea developed in Section 3.1.

\subsubsection{Physical Conditions in the [O III] Shell}

The average hydrogen number density of the [O III] shell, $n$, can be estimated from the $\mathrm{H} \alpha$ surface brightness 
(photons $\mathrm{cm}^{2} \mathrm{~s}^{-1} \mathrm{sr}^{-1}$ ) given by

$$
S(\mathrm{H} \alpha)=x_{\mathrm{e}} \alpha_{\mathrm{eff}} n^{2} L / 4 \pi,
$$

where $L$ is the path length of the line of sight through the shell and $x_{\mathrm{e}}$ is the electron fraction. Both hydrogen and helium are assumed to be singly ionized, with $\mathrm{He} / \mathrm{H}=0.0977$ (Esteban et al. 1998), so that $x_{\mathrm{e}}=1.0977$. The effective line recombination coefficient $\alpha_{\text {eff }}$ has a very weak density dependence and can be approximated in the Case B limit as $1.16 \times 10^{-13} T_{4}^{-1} \mathrm{~cm}^{3} \mathrm{~s}^{-1}$ (Osterbrock \& Ferland 2006), where $T_{4}=T / 10^{4} \mathrm{~K}$. For a spherical shell of radius $R$ and fractional thickness $\Delta \ll 1$, the maximum path length through the shell is $L \simeq 2 \Delta^{1 / 2} R$. The observed radius and thickness of the [O III] shell vary with position angle (P.A.) by about $50 \%$ but we will take $R=2 \times 10^{17} \mathrm{~cm}, \Delta=0.28$ as typical of the well-defined northern arm of the shell, so that $L \simeq R$.

The surface brightness of the shell is most easily estimated from the sharp brightness jump at the inner edge of its limbbrightened northern arm. Using WFPC2 images (O'Dell \& Wong 1996), flux-calibrated following O'Dell \& Doi (1999), and extinction-corrected following O'Dell \& Yusef-Zadeh (2000), we find $S(\mathrm{H} \alpha)=2.85 \times 10^{10}$ photons $\mathrm{cm}^{2} \mathrm{~s}^{-1} \mathrm{sr}^{-1}$, which represents about $25 \%$ of the total nebular surface brightness at the shell position. We also find $[\mathrm{O}$ III $] / \mathrm{H} \alpha=1.62$ and no evidence of any [N $\left.\mathrm{NI}_{\mathrm{II}}\right]$ emission from the shell ([N $\left.\mathrm{II}\right] / \mathrm{H} \alpha<$ $0.01)$.

For the shell temperature, we use the results of Mesa-Delgado et al. (2008) who find $T \simeq 8300 \mathrm{~K}$ at the position of the shell from the ratio of the $4363 \AA$ and $5007 \AA$ [O III] lines (the upper-right panel of their Figure 6). This temperature represents an average of the emission from the shell and the background nebula at that position, but there is no evidence for any significant jump in $T$ at the inner edge of the shell, so we can be confident in using it for the shell temperature, giving an effective recombination coefficient of $1.36 \times 10^{-13} \mathrm{~cm}^{3} \mathrm{~s}^{-1}$.

Combining all of the above, we find a shell-emission measure of $\mathrm{EM}=x_{\mathrm{e}} n^{2} L=2.56 \times 10^{24} \mathrm{~cm}^{-5}$ and hence a density $n=3400 \mathrm{~cm}^{-3}$. The ionizing flux at the position of the shell is $\Phi_{\mathrm{H}}=Q_{\mathrm{H}} / 4 \pi R^{2} \simeq 3.6 \times 10^{13} \mathrm{~cm}^{-2} \mathrm{~s}^{-1}$, where $Q_{\mathrm{H}}$ is the ionizing luminosity of $\theta^{1}$ Ori $\mathrm{C}$, assumed to be $1.8 \times 10^{49} \mathrm{~s}^{-1}$ (Henney et al. 2005).

The ionization parameter in the shell $\left(\Phi_{\mathrm{H}} / n c=0.35\right)$ is very large when compared with typical values for the nebula as a whole (0.01-0.02). Therefore, if dust grains are not significantly underabundant, the grains will dominate the ultraviolet opacity of the shell. Assuming a dust extinction cross section of $\sigma_{\mathrm{d}}=10^{-21} \mathrm{~cm}^{2}$ per $\mathrm{H}$ nucleon (Baldwin et al. 1991), the optical depth of grains in the shell to ionizing radiation is $n \sigma_{\mathrm{d}} R \Delta \simeq 0.2$. For comparison, the neutral hydrogen optical depth is much smaller: $\alpha_{\mathrm{B}} \mathrm{EM} / \Phi_{\mathrm{H}} \simeq 0.022$. Thus, the shell is optically thin to EUV radiation, which, combined with the high-ionization parameter, means that low-ionization lines such as [N II] are expected to be very weak. Indeed, no detectable [N II] emission is observed from the shell.

\subsection{Space Motion of $\theta^{1}$ Ori C}

In spite of its brightness, it has been difficult to determine the 3D space motion of $\theta^{1}$ Ori $\mathrm{C}$, the dominant ionizing star. This is true for both the tangential and radial velocity components that are needed for deriving this motion.

The brightness relative to the other cluster members presents particular challenges in determining the tangential velocity. The most accurate study is that of van Altena et al. (1988), who determined that $\theta^{1}$ Ori $\mathrm{C}$ was moving at $4.8 \pm 0.5 \mathrm{~km} \mathrm{~s}^{-1}$ toward P.A. $142^{\circ}$ and that this was significantly larger than the dispersion value of $1.5 \pm 0.1 \mathrm{~km} \mathrm{~s}^{-1}$ found for the other cluster stars ( 49 cluster members in the sample). Jones \& Walker (1988) did not include the brightest members of the ONC in their astrometric study, but did find a velocity dispersion of $1.5 \pm 0.7 \mathrm{~km} \mathrm{~s}^{-1}$ for the brighter stars in their study. Tan (2004) interpreted this high tangential velocity away from the direction of the imbedded BN-KL cluster stars as evidence that $\theta^{1}$ Ori $\mathrm{C}$ shares a point of origin with the radio source BecklinNeugebauer $(\mathrm{BN})$, which is moving in the opposite direction at a high velocity $\left(38 \mathrm{~km} \mathrm{~s}^{-1}\right.$ at P.A. $\left.=322^{\circ}\right)$, and that the present tangential velocity would have placed the $\mathrm{BN}$ object in the vicinity of the Trapezium about 4000 years ago. However, subsequent studies (Rodríguez et al. 2005; Gómez et al. 2005, 2008) of the radio sources within the $\mathrm{BN}-\mathrm{KL}$ region show that three radio sources in the center of the $\mathrm{BN}-\mathrm{KL}$ region are moving away from a common center with a timescale of 500 years and that the BN-KL source I used for reference by Tan (2004) was one of the moving objects. Gómez et al. (2005) found that BN was moving toward the northwest at $24 \mathrm{~km} \mathrm{~s}^{-1}$ in the Orion cluster rest frame. The separation of $\theta^{1}$ Ori $C$ and the dynamic center of BN-KL is $63^{\prime \prime}$, which means that a backward projection of $\theta^{1}$ Ori C's tangential motion as determined by van Altena et al. (1988) would cross that position in about 28,000 years. Even if it is moving away from the BN-KL center, this timescale indicates that it is unlikely that $\theta^{1}$ Ori $\mathrm{C}$ is a runaway object from the $\mathrm{BN}-\mathrm{KL}$ region event that produced the three escaping radio sources and the bipolar outflow with an upper limit age of 1000 years (Doi et al. 2002) that has produced the well-known $\mathrm{H}_{2}$ fingers.

Even the modest anomalous tangential velocity of $4.8 \mathrm{~km} \mathrm{~s}^{-1}$ may be too high. The data of van Altena et al. (1988) indicate that $\theta^{1}$ Ori $\mathrm{C}$ and $\theta^{1}$ Ori $\mathrm{B}$ are moving in opposite directions at about 3.5 mas year ${ }^{-1}$. In a compilation of visual astrometric measurements of the relative positions of the Trapezium stars, Allen et al. (1974) present results for these two stars over a time interval of 125 years, during which time the stars would have separated by 0.43 , but there is no detectable change to an accuracy of about 0.15 .

Determination of the radial velocity of $\theta^{1}$ Ori $\mathrm{C}$ is also difficult (O'Dell 2001b; Vitrichenko 2002). In this case the brightness of the star aids the collection of high-velocity resolution spectra, but the star is complex, being an obliquerotation magnetic star which shows a bewildering pattern of periodic and erratic spectral changes. It has a resolved early-type star companion. The properties of the system are summarized in Muench et al. (2008). Utilizing only spectroscopic radialvelocity data, Vitrichenko (2002) determined a systemic radial velocity of $10.9 \pm 2 \mathrm{~km} \mathrm{~s}^{-1}$ and by combining spectroscopic and astrometric (Kraus et al. 2007; Patience et al. 2008) data, Stahl et al. (2008) have determined the systemic radial velocity of $\theta^{1}$ Ori $\mathrm{C}$ as $13 \mathrm{~km} \mathrm{~s}^{-1}$, which is quite different from that of the other Trapezium stars and the lower mass ONC members, as summarized in Table 1, and indicating that it is moving rapidly away from the OMC and its host star cluster (relative velocity about $13 \mathrm{~km} \mathrm{~s}^{-1}$ ). In Section 3.4, we examine the effect that such a rapid motion for $\theta^{1}$ Ori $C$ would have on the present structure and evolution of the Orion Nebula.

This special characteristic (high spatial velocity) may not be all that unexpected. Stone (1991) concludes that in general $40 \%$ of all $\mathrm{O}$ stars are runaways, although only one in $10 \mathrm{OB}$ stars has 
a known binary companion. There is an observational selection effect operating in the ONC that favors detection of blueshifted radial velocities. A redshifted runaway would be moving into the obscured portion of the OMC and we would not be seeing the photoionized Orion Nebula at visual wavelengths. This means that if we are going to detect an anomalous radial velocity, it is going to be a blueshift. The difference in radial velocity with respect to the other Trapezium stars would mean that either this juxtaposition of massive stars is coincidental or perhaps that dynamical interactions with the other stars has produced $\theta^{1}$ Ori C's anomalous radial velocity.

Certainly, runaway stars are expected from a grouping like the Trapezium (Pflamm-Altenburg \& Kroupa 2006) and have been created previously in the ONC. The divergence from Orion of the two high-velocity stars AE Aur and $\mu$ Col has been known for over a half century (Blaauw \& Morgan 1954). Calculations of the trajectories of these stars and ८ Ori (Hoogerwerf et al. 2001) indicate that they all originated about 2.5 million years ago from a position now occupied by the ONC. This is not to argue that these stars arose in the Trapezium grouping, however, it is evidence that there was an earlier epoch of massive star formation in this vicinity. Given today's presence of three centers of star formation (the ONC, BN-KL, and Orion-S), it is not hard to accept that there was at least one earlier similar center. Likewise, the earlier creation of runaways argues that this could have again occurred and $\theta^{1}$ Ori $\mathrm{C}$ is the product.

\section{DISCUSSION}

The new features presented in Section 2 have caused us to reassess several major properties of the Orion Nebula. In Section 3.1, we consider the effects of the high-velocity wind from $\theta^{1}$ Ori C. In Section 3.2, we present arguments for the Orion-S feature being a separate cloud within the ionized cavity of the nebula, and in Section 3.3 we present a model consistent with it producing $\mathrm{H}_{2} \mathrm{CO}$ in absorption. We conclude with a discussion of how our view of the nebula would be altered if $\theta^{1}$ Ori C has a large blueshifted velocity with respect to the other members of the ONC.

\subsection{The Effects of the High-Velocity Wind from $\theta^{1}$ Ori C}

The presence of a high-velocity SW from $\theta^{1}$ Ori $\mathrm{C}$ is characteristic of early spectral type stars and will certainly affect the conditions of the nearby nebular gas. In this section we address the nature of the wind, its expected interactions, and compare the expectations with what is observed.

The radiatively driven SW is modified by the star's strong dipolar magnetic field (Donati et al. 2002), leading to a very anisotropic outflow (Smith \& Fullerton 2005). However, the large inclination between the magnetic and rotation axes (Wade et al. 2006) means that much of this anisotropy is smoothed out over the 15.422 day rotational period (Stahl et al. 1993, 1996), so that it is reasonable to assume that the wind is approximately isotropic when considering its interaction with the Orion Nebula. Angle-averaged values for the mass-loss rate and terminal velocity of $M_{\mathrm{w}}=4 \times 10^{-7} M_{\odot} \mathrm{yr}^{-1}$ and $V_{\mathrm{w}}=1400 \mathrm{~km} \mathrm{~s}^{-1}$ have been determined from modeling the $\mathrm{X}$-ray emission from the base of the wind (Gagné et al. 2005).

A totally independent measurement of the strength of the fast $\mathrm{SW}$ is provided by the $[\mathrm{O} \mathrm{III}] / \mathrm{MIR}$ arcs seen in front of the closest proplyds to $\theta^{1}$ Ori C (Hayward et al. 1994; Bally et al. 1998; Robberto et al. 2005; Smith et al. 2005), which are interpreted as the stationary stand-off bowshocks that result from the interaction of the proplyd photoevaporation flow (PF) with the SW (García-Arredondo et al. 2001). The position of these bowshocks is determined by the balance between the ram pressures of the proplyd outflow and the fast wind from $\theta^{1}$ Ori C, so that they can be used as probes of $\dot{M}_{\mathrm{w}} V_{\mathrm{w}}$ so long as the proplyd parameters can be determined with sufficient precision. Application to the best-studied proplyd 167-317 (García-Arredondo et al. 2001, 2002; Henney et al. 2002) yields a value of $\left(\dot{M}_{\mathrm{w}} / 10^{-7} M_{\odot} \mathrm{yr}^{-1}\right)\left(V_{\mathrm{w}} / 1000 \mathrm{~km} \mathrm{~s}^{-1}\right)=4.6 \pm 1.4$, which is consistent with the Gagné et al. (2005) values given above.

\subsubsection{Theoretical Predictions of the Wind/Nebula Interaction}

The interaction of the fast wind from $\theta^{1}$ Ori $\mathrm{C}$ with its surroundings differs from the standard spherical hot-shocked wind bubble scenario (see e.g., Dyson \& Williams 1997) due to the effects of geometry, density gradients, mass loading from embedded sources, and possibly thermal conduction. The different wind interaction regions are summarized in Figure 4, which synthesizes findings from many recent theoretical and observational studies. Each region is discussed further below, starting from the inside. We concentrate on the inner parts of the nebular that are relevant to the observational material presented in this paper, leaving aside considerations of the EON from which X-ray evidence of SW interaction has recently been reported (Güdel et al. 2008).

Mass-loading zone. Mass loading of an SW by embedded sources lowers the SW velocity of the unshocked wind and consequently reduces the temperature and modifies the cooling properties in the hot-shocked wind region (Hartquist et al. 1986). In the Orion Nebula, the sources of mass are the photoevaporated flows from the close-in proplyds (PB in the figure) and the SWs from the other Trapezium stars (SB). Of these sources, the proplyds are the most important (García-Arredondo et al. 2001), since the mass-loss rate from each proplyd is of the same order as $\dot{M}_{\mathrm{w}}$ (Henney \& O'Dell 1999; Henney et al. 2002), whereas the most massive of the other Trapezium stars are early B-type, with winds at least an order of magnitude weaker than that of $\theta^{1}$ Ori $\mathrm{C}$ (MS in the figure). Unfortunately, the efficiency of the mixing between the SW and the proplyd PFs is poorly constrained (García-Arredondo et al. 2002), leading to large uncertainties in the degree of deceleration of the wind. However, as long as the mixed wind remains highly supersonic, its momentum $M_{\mathrm{w}} V_{\mathrm{w}}$ is conserved.

Shocked wind. When this mass-loaded, but still supersonic, wind interacts with the nebula, a two-shock flow pattern is formed (Pikel'ner 1968; Dyson \& de Vries 1972): an outer shock that accelerates the nebular gas and an inner shock (the blue doubledashed line in Figure 4) that decelerates the SW. The two shocked regions are separated by a contact discontinuity (blue dotted line), across which the pressure is continuous. If the inner wind shock is nonradiative (as is usually the case), the shocked SW will have a temperature $T / 10^{6} \mathrm{~K} \simeq 0.15\left(V_{\mathrm{w}} / 100 \mathrm{~km} \mathrm{~s}^{-1}\right)^{2}$ and a thermal pressure roughly equal to the ram pressure at the inner shock.

Large-scale density gradients in the adjoining MC mean that the inner shock is much closer to the star in the cloud direction (upper-left in Figure 4) than in the opposing direction (offpicture to the lower-right), leading to a higher pressure of the shocked SW in the cloud-facing direction, which therefore flows toward the low-density end of the bubble (Arthur \& Hoare 2006) in a transonic wind exhaust (WE in Figure 4). A simple steady- 


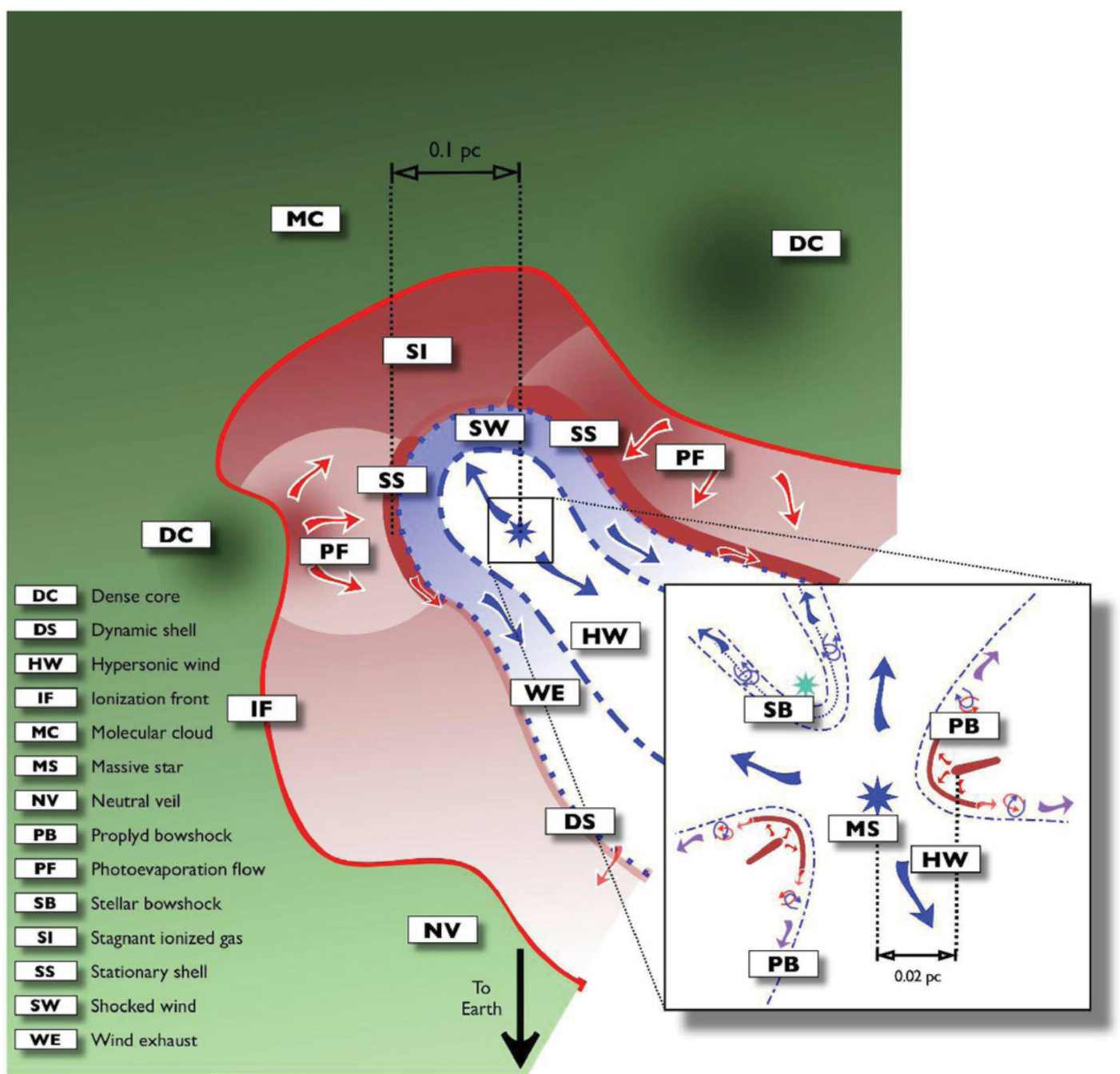

Figure 4. Schematic cross section through the inner Orion Nebula, showing the different zones of SW interaction. Blue indicates SW material, red indicates photoionized nebular material, and green indicates atomic/molecular gas, with darker shading corresponding to qualitatively higher density within each region. Arrows show the approximate direction of gas flows, with sizes that roughly scale with the momentum flux. Shocks in the SW are shown by short-long-dashed lines, while the contact discontinuities are shown by dotted lines. The drawing does not represent any particular cut through the nebula, and is not exactly to scale, although the sizes in parsecs of some major features are marked. The observer is located off the bottom of the picture. The inset box shows a zoom of the central region.

state model then gives the radius of the wind shock as 0.73 times the radius of the contact discontinuity.

Shocked nebula. In the early evolution of an $\mathrm{H}_{\text {II }}$ region, the ionization front is likely to be trapped inside the dense shell swept up by the outer wind-driven shock (García-Segura \& Franco 1996; Arthur \& Hoare 2006). The density in the shell decreases with time, so that the ionization front eventually escapes from the shell and drives a low-velocity neutral shock ahead of itself into the molecular gas (Arthur 2007). After this time, the outer wind-driven shock will be interacting with photoionized nebular gas, as shown in Figure 4. If this gas is static, with a constant hydrogen number density $n \mathrm{~cm}^{-3}$, then the outer shock will degenerate into a sound wave after $\simeq 1.3 / n^{1 / 2}$ million years (Dyson \& Williams 1997), leading to dissipation of the shocked nebular shell, after which one will have approximate static pressure equilibrium between the $\mathrm{H}$ II region and the shocked wind. In the cloud-facing direction, where the ionized density is $n=10^{3}-10^{4} \mathrm{~cm}^{-3}$, this will occur on a timescale much shorter than the age of the nebula, so that stagnant zones of ionized gas (SI in Figure 4) can be confined by the wind (Arthur \& Hoare 2006). In the direction away from the cloud, the ionized density is much lower, $n<100 \mathrm{~cm}^{-3}$
(Felli et al. 1993), so that one may still have a dynamic shell (DS) of swept-up $\mathrm{H}$ II region that moves outward at a few tens of $\mathrm{km} \mathrm{s}^{-1}$.

Additional structures will arise due to the internal dynamics of the ionized gas in the $\mathrm{H}$ II region. Turbulence and gravitational collapse in MCs (Vázquez-Semadeni et al. 2005) produce density concentrations on multiple scales (idealized as dense cores, DC, in Figure 4), which retard the progress of the ionization front. In regions where the ionization front is locally convex or flat, PFs will develop (Henney et al. 2005; Mellema et al. 2006), which reach mildly supersonic velocities of 20$40 \mathrm{~km} \mathrm{~s}^{-1}$ (Dyson 1968; Bertoldi 1989). Where one of these PFs interacts with the SW bubble, a stationary shell (SS) of shocked ionized gas will form at a position where the ram pressure of the PF balances the thermal pressure of the shocked wind.

\subsubsection{Observational Evidence for the Effects of the Wind on the Ionized Nebula}

As reported in Section 2.6.1, we have found an incomplete high-ionization shell in the inner Orion Nebula, which may be related to the action of the SW from $\theta^{1}$ Ori C. Since the shell has both an inner and outer boundary (Figure 3), it would 
correspond to an SS in the context of the above discussion (SS in Figure 4). This means that the kinematics of the shell is likely to be unremarkable, as compared with the kinematics of the general [O III] emission from the nebula. Just as with the rest of the nebular gas, the gas in the shell will find it easier to move in the direction away from the MC and so should be slightly blueshifted on average compared with the systemic velocity of the stars and molecular gas. Inspection of the [O III] velocity cubes of Doi et al. (2004) shows that the line profiles from the shell are, indeed, very typical of the nebula as a whole.

The inner boundary of the shell corresponds to the contact discontinuity with the shocked wind bubble, so the shell's thermal pressure should equal that of the shocked wind. From the physical parameters derived from observations in Section 2.6.2, one finds a shell pressure of $P_{\mathrm{sh}} / k=\left(1+x_{\mathrm{e}}\right) n T \simeq 5.9 \times$ $10^{7} \mathrm{~cm}^{-3} \mathrm{~K}$. The bubble pressure will be equal to the wind ram pressure at the inner wind shock: $P_{\mathrm{bub}} / k=\dot{M}_{\mathrm{w}} V_{\mathrm{w}} / 4 \pi R_{\text {in }}^{2} k \simeq$ $7.84 \times 10^{7} \mathrm{~cm}^{-3} \mathrm{~K}$, where we have assumed that the inner shock radius is $R_{\text {in }}=0.73 R$ (see above) and that the wind momentum loss rate $\dot{M}_{\mathrm{w}} V_{\mathrm{w}}$ is the proplyd-derived value discussed in Section 3.1. Given the uncertainties in their derivation, these pressures are indistinguishably the same, providing strong evidence that the [O III] shell has been compressed by the SW.

The Red Bay (García-Díaz \& Henney 2007) is a region in which the high-ionization gas, as traced by [S $\mathrm{III}]$ and [O III] lines, has a velocity similar to that of the $\mathrm{MC}\left(V_{\odot}=25-\right.$ $\left.30 \mathrm{~km} \mathrm{~s}^{-1}\right)$, rather than the more blueshifted velocities $\left(V_{\odot}<\right.$ $25 \mathrm{~km} \mathrm{~s}^{-1}$ ) found elsewhere in the nebula. The region of relatively redshifted velocities starts at the Trapezium and extends in a broad strip $\left(\simeq 75^{\prime \prime} \times 25^{\prime \prime}\right)$ to the east-southeast, which can be best appreciated as a light-colored region in the [O III] mean velocity map (Figure 13 of García-Díaz et al. 2008). ${ }^{7}$ The Red Bay is also associated with a region of lower-thanaverage [O III] surface brightness.

Comparison of the emission measure and electron density in this region (García-Díaz \& Henney 2007) shows that it is not a thin shell seen face-on, but has a thickness along the line of sight $\simeq 10^{18} \mathrm{~cm}$, which is roughly twice its extent in the plane of the sky. We therefore suggest that the Red Bay may correspond to a stagnant zone of ionized gas (SI in Figure 4), which is trapped between a concave ionization front, the SW bubble, and the PFs from molecular concentrations such as the bright bar.

\subsection{The 3D Structure of the Orion-S Region}

The several pieces of published and newly derived evidence require that we revise the accepted picture that Orion-S is simply a local rise in the MIF caused by dense underlying material in the OMC. Certainly, this is a region of enhanced density as determined from $\mathrm{H}^{13} \mathrm{CO}^{+}$observations. The recently published $21^{\prime \prime}$ resolution $\mathrm{H}^{13} \mathrm{CO}^{+}$maps that include the Huygens region (Ikeda et al. 2007) show that the most massive cores lie about $30^{\prime \prime}$ north of BN-KL and within Orion-S, but these observations cannot discriminate between cores that lie within a foreground optically thick feature and something that lies behind the MIF and within the main OMC filament. However, the presence of $\mathrm{H}_{2} \mathrm{CO}$ absorption in the radio continuum (as discussed in Section 2.3) clearly places a high density, very optically thick object in front of an ionized volume and this object could also be

\footnotetext{
7 As originally drawn in Figure 7 of García-Díaz \& Henney (2007), based on relatively low signal-to-noise [S III] observations, the Red Bay was broader, extending somewhat north of the Trapezium. However, this interpretation is not supported by the better-quality [O III] data.
}

the source of the peak in rotationally excited $\mathrm{CO}$ emission that lies between the $\mathrm{H}_{2} \mathrm{CO}$ absorption feature and the Trapezium (Wilson et al. 2001). The disparity in the extinction derived from radio-to-optical and optical line ratios (as discussed in Section 2.4) further confirms that the optical features we see lie in the foreground of an ionized volume. The low ionization seen on the northeast side of Orion-S is most easily explained as an inclined face analogous to the escarpment causing the bright bar, but not as tilted. Otherwise it is impossible to explain the [N II] surface brightness this close to the ionizing star.

There are arguments that the foreground Orion-S Cloud hosts the embedded stars that are the sources of several optical features and outflows. A dark rectangular form lies almost exactly on the redshifted $\mathrm{SiO}$ outflow and the IR source 139-357. It is not certain which (or if both) of these two features is associated, but an association of an optical feature with either places that feature in the foreground, rather than something within the OMC. There is a suggestive notch in the $\mathrm{H}_{2} \mathrm{CO}$ absorption contour coincident with both the blueshifted outflows in $\mathrm{SiO}$ and $\mathrm{CO}$, so that one or both of these must be in the foreground feature. The presence of high-ionization, high tangential velocity features symmetric with the Dark Arc (O'Dell \& Yusef-Zadeh 2000; O'Dell et al. 2008) indicates that it is also part of the foreground structure.

The $\mathrm{H}_{2} \mathrm{CO}$ absorption indicates very high optical depths in the near ultraviolet and it is unlikely that the obscuration by the foreground feature stops at the lowest contour of detection of $\mathrm{H}_{2} \mathrm{CO}$ absorption. This high extinction allows us to comment on the location of the sources that give rise to optical outflows. The sources of the large-scale optical outflows have been isolated to a small region shown as the dark dashed-line ellipse in Figure 1 (O'Dell et al. 2008). These outflows are all blueshifted. The eastoriented HH 529 shocks are high ionization and are seen very close to the source, indicating that this flow emerges quickly from behind the local ionization front. The west-oriented $\mathrm{HH}$ 269 shocks and jet also emerge close to their sources and are seen visually, arguing that they too originate in the foreground Orion$\mathrm{S}$ Cloud. The emergence further from the source region (O'Dell et al. 2008) of the high-velocity gas driving the northwestoriented HH 202 and the southeast-oriented HH 203 and HH 204 could either indicate that their sources are more deeply imbedded in the Orion-S Cloud or lie within the background OMC. The latter interpretation would imply that the lack of visibility near the source region is due to the high optical extinction of the Orion-S Cloud, but it seems more likely that all the sources lie at a similar distance along the line of sight.

There is a question about the location of the strongest IR and radio sources in this region: 137-408 (CS 3 in earlier, lowresolution studies) and 134-411 (FIR 4 in earlier, low-resolution studies). The symmetry axis of the nearby HH 530 shocks points to either of these sources and the proximity of such an optical feature argues that its source lies in the foreground Orion-S Cloud.

The far-IR emission from dust heated by embedded strong IR sources (Houde et al. 2004) indicates (Figure 1) a peak in the vicinity of $\mathrm{BN}-\mathrm{KL}$ and a weaker elongated peak in the Orion-S feature. The northerly extension of this southern feature coincides with the source region for the optical outflows and the southerly extension coincides with the 137-408 and 134-411 sources. Unfortunately, this does not tell us if the southerly extension belongs to the foreground feature or lies within the OMC.

The most likely geometry for this region is shown in Figure 5. In this model, we have placed the Orion-S Cloud far enough 


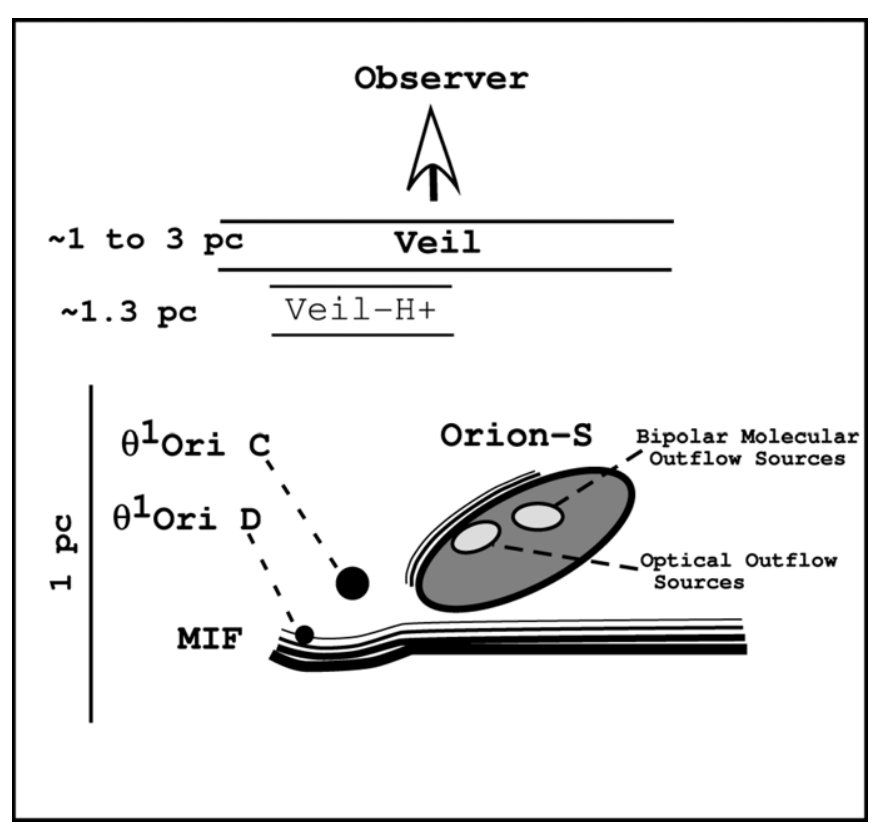

Figure 5. This sketch shows a southwest cross section passing through the Trapezium and Orion-S. The MIF is depicted as flat behind Orion-S only because of a lack of information as to its true shape there. Although dimensions are shown, the uncertainty in the exact positions means that the figure is not drawn to scale.

into the foreground that the MIF extends continuously behind it. The exact placement of the Orion-S Cloud in the foreground is uncertain, but it must be close to the distance of $\theta^{1}$ Ori $\mathrm{C}$ in front of the local MIF because if it were much further from the observer it could not be distinguished from the MIF. In the same way that the bright bar probably represents where the ionization front of the nebula encounters a long high-density condensation within the host OMC, it is likely that the Orion-S Cloud was a high-density feature within the OMC, which has been isolated as the ionization front progressed into the host MC. This conclusion is compatible with the fact that the velocity of the $\mathrm{H}_{2} \mathrm{CO}$ extinction feature $\left(V_{\mathrm{H}_{2} \mathrm{CO}}=28.6 \pm 2\right)$ is essentially the same as the host $\mathrm{MC}\left(V_{\mathrm{OMC}}=25.9 \pm 1.7\right)$.

\subsection{Chemistry of the Orion-S Cloud}

Distinctly different heating processes are important in various regions of the Orion environment. Starlight is the most important heating/ionization process in the bright $\mathrm{H}^{+}$region and its associated PDR. Wind-driven shocks are responsible for much of the geometry and emission of the BN object. Cosmic rays and heating by dissipative MHD waves may be important in deeper regions of OMC1. Given the assortment of possible dynamical and microphysical processes involved, two approaches to numerical simulations of the ISM can be taken. Hydrodynamics codes follow the motions of the gas but must compromise on the microphysics to solve the problem on today's computers. A microphysics code (like Cloudy, which we use here) will not compromise on the microphysics but must do something simple for the structure of the cloud. Atomic processes are usually much faster than dynamical timescales so the physics that determines the spectrum is often not affected by the dynamics and a static geometry can be assumed.

In this section, we wish to understand several overall features of the Orion-S Cloud via a simulation of the ionization, thermal, and chemical state of the cloud. The primary question we want to answer is "can we explain the presence of $\mathrm{H}_{2} \mathrm{CO}$ in absorption?" Furthermore, can we do so in the context of other molecules seen in emission and the geometrical constraints imposed by the projected size of Orion-S? There are estimates of the gas column density, particle density, temperature, and gas pressure (which is surprisingly high). Can starlight from the Trapezium cluster account for these general properties, or are other processes at play? Here, we test whether starlight from the Trapezium cluster can account for several of the observed properties of Orion-S.

\subsubsection{Observational Constraints and Geometry}

The most important constraints to modeling the Orion-S Cloud are the $\mathrm{H}_{2} \mathrm{CO}$ absorption measurements, the total column density, and the projected size of Orion-S. In cold, dark MCs, chemical models, which include the effects of accretion of molecules onto grain surfaces (Hasegawa et al. 1992; Hasegawa \& Herbst 1993), show that $\mathrm{H}_{2} \mathrm{CO}$ will reside almost exclusively on the surface of grains (henceforth "freeze out") at temperatures at or below $10 \mathrm{~K}$. A temperature of $10 \mathrm{~K}$ is also consistent with the ionized and molecular gas in Orion-S being in gas-pressure equilibrium (see below). However, since $\mathrm{H}_{2} \mathrm{CO}$ is observed in the gas phase in Orion-S, the temperature must be higher, such that the rate of evaporation of $\mathrm{H}_{2} \mathrm{CO}$ from grain surfaces, $k_{\text {evap }}=1.7 \times 10^{12} \exp \left(-1760 / T_{\mathrm{d}}\right)$, must be large enough to produce a significant gas-phase abundance of $\mathrm{H}_{2} \mathrm{CO}$. Figure 6 shows that this rate will be fast $\left(>10^{-10} \mathrm{~s}^{-1}\right.$ ) for $T_{\mathrm{d}}>34 \mathrm{~K}$. Therefore, $\mathrm{H}_{2} \mathrm{CO}$ observations imply that the dust temperature (which should be nearly in equilibrium with the gas temperature for deeper regions of the cloud) needs to be roughly $30 \mathrm{~K}$ or higher in order to explain the presence of $\mathrm{H}_{2} \mathrm{CO}$. The total $\mathrm{H}$ column density is given in McMullun et al. (1993), who through 1.3 and $3.1 \mathrm{~mm}$ dust continuum emission found $N(\mathrm{H}) \sim 4 \times 10^{24} \mathrm{~cm}^{-2}$ for the region $12^{\prime \prime} \mathrm{N}$ of the $3.1 \mathrm{~mm}$ continuum peak, corresponding to a region they referred to as the local quiescent cloud. The projected separation of the Trapezium stars from Orion-S is $2.2 \times 10^{17} \mathrm{~cm}$, and the projected diameter of the cloud is $\sim 2.8 \times 10^{17} \mathrm{~cm}$. Assuming an approximately spherical shape then gives a total cloud mass of $280 M_{\odot}$ (McMullun et al. 1993).

The close proximity of Orion-S to the Trapezium means there must be an $\mathrm{H}^{+}$region on the side of Orion-S facing the Trapezium. Optical emission lines suggest that it has a density of $\sim 6000 \mathrm{~cm}^{-3}$ (Pogge et al. 1992; García-Díaz \& Henney 2007 ), corresponding to a gas pressure of $P / k=2 n T=$ $1.08 \times 10^{8} \mathrm{~cm}^{-3} \mathrm{~K}$, assuming a gas temperature of $8500 \mathrm{~K}$. For the assumed thickness of Orion-S, and the above estimate of $N(\mathrm{H})$, the density deep in the MC of Orion-S is $3 \times 10^{7} \mathrm{~cm}^{-3}$. If the environment were in gas-pressure equilibrium, then the corresponding MC pressure, $0.5 n T=2 \times 10^{7} T \mathrm{~cm}^{-3} \mathrm{~K}$, would correspond to a temperature of $7 \mathrm{~K}$. However, as noted above, this is inconsistent with the presence of gas-phase $\mathrm{H}_{2} \mathrm{CO}$, indicating that the gas pressure in the molecular gas must be several times higher than that in the ionized gas.

There are at least four different factors that may account for this overpressure of the molecular gas:

1. If the ionization front on the surface of the cloud is D-critical, then the transonic ionized flow from the front contributes an additional ram pressure term, which is roughly equal to the thermal pressure. On the neutral/ molecular side, the net flow toward the front is highly subsonic, so the ram pressure term there is negligible. In a steady-state equilibrium, this gives a molecular gas pressure twice that of the ionized gas. 


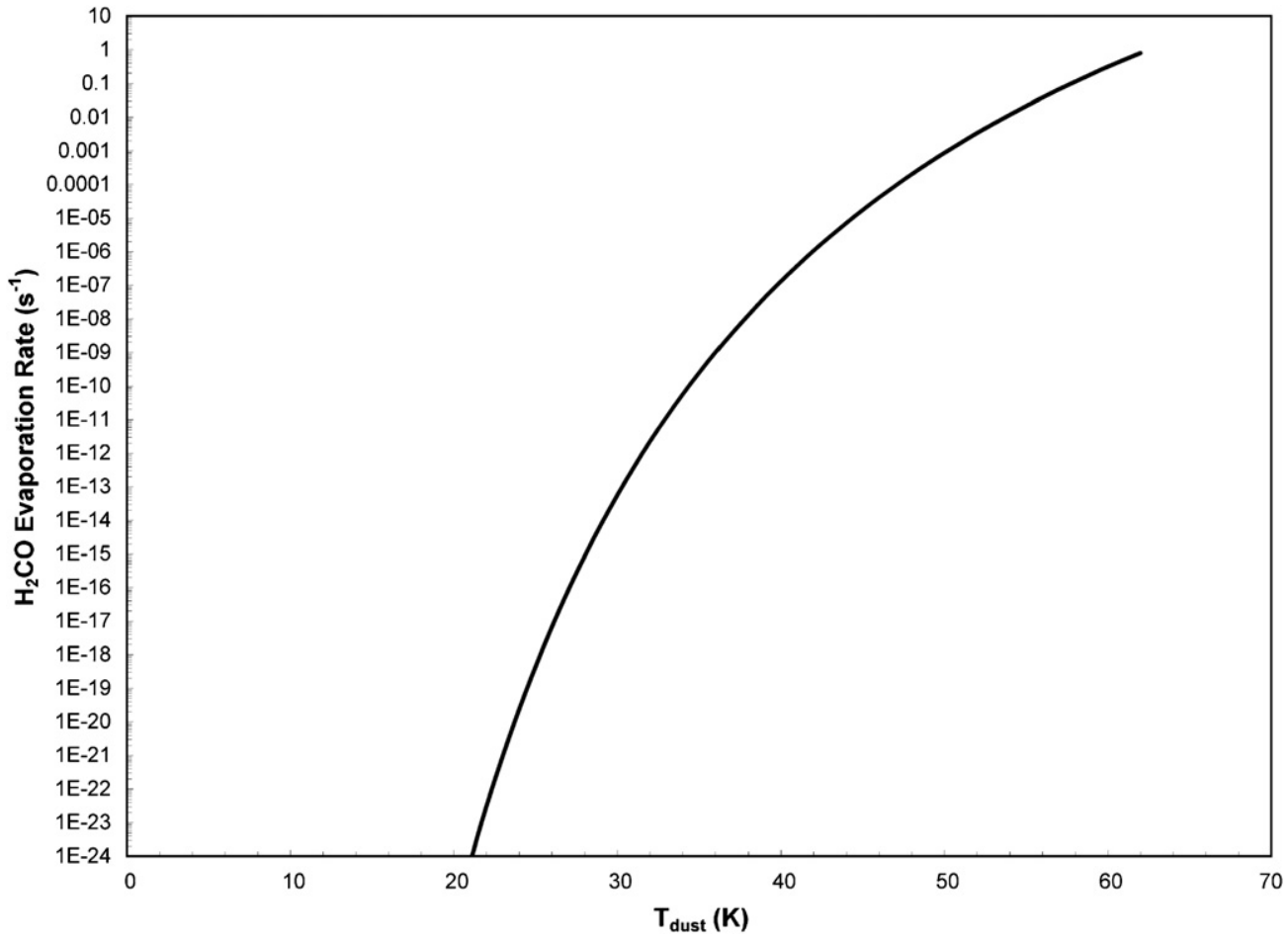

Figure 6. $\mathrm{H}_{2} \mathrm{CO}$ evaporation rate as a function of grain temperature.

2. Absorption of the momentum of the stellar photons produces an outwardly directed effective gravity. In a steadystate configuration, in which the molecular gas is forced to remain at rest with respect to the star, this must be balanced by a pressure gradient between the optically thin and optically thick portions of the cloud.

3. If the cloud is self-gravitating and hydrostatic, then the deeper gravitational potential in the center of the cloud will produce a higher pressure for the molecular gas there than for the ionized gas at the cloud surface.

4. If the cloud is a transient phenomenon, formed from the dynamic interaction of converging flows, then there is no reason to expect it to be in pressure equilibrium at all.

On the other hand, there are two factors that would tend to work in the other direction:

5. Magnetic fields tend to be relatively weak in ionized nebulae (Heiles et al. 1981), with magnetic pressure smaller than the gas pressure. In MCs, on the other hand the reverse is frequently true, with the magnetic pressure typically exceeding the gas pressure by a factor of 10 or more (Crutcher 1999).

6. Turbulent internal motions in MCs are frequently supersonic, with turbulent ram pressure approximately equal to the magnetic pressure.

Therefore, the increase in gas pressure, when going from ionized to molecular gas, indicates that one or more of the first four factors must outweigh factors 5 and 6 .

\subsubsection{An Equilibrium Cloudy Model of Orion-S}

We perform calculations with version 8.0 of the spectral synthesis code Cloudy, described by Ferland et al. (1998). Extensions to PDR physics and chemistry are described by
Abel et al. (2005, 2008). The gas-phase composition and dust properties of the Orion environment are well known and are given in Abel et al. (2004a).

Our choice of ionizing continuum is characteristic of the dominant source of ionizing photons, the $\mathrm{O} 7$ star $\theta^{1}$ Ori C. We assume a Kurucz stellar atmosphere with a temperature of $39,600 \mathrm{~K}$, and $Q(H)=1.7 \times 10^{49}$ photons s $^{-1}$. We include $\mathrm{X}$-ray emission from the $\mathrm{SW}$ (see Section 3.1), modeled as a $10^{6} \mathrm{~K}$ bremsstrahlung component, although this does not affect the results presented below.

For simplicity, we consider a time-independent, magnetostatic equilibrium for the Orion-S Cloud, as has been recently employed to account for the structure and observed magnetic field in M17 (Pellegrini et al. 2007). The current version of Cloudy does not treat self-gravity, and we also ignore gas motions and time-dependent effects, so the model cannot directly address factors 1,2 , or 4 , listed in the previous section. In the model, the total pressure increases as one proceeds into the cloud in such a way that the local pressure gradient exactly counterbalances the acceleration due to absorption of radiation. The total pressure is the sum of the thermal, magnetic, turbulent, and trapped resonance line radiation contributions. For simplicity, we assume that the turbulent pressure is in equipartition with the magnetic field and that the magnetic field is tangled on small scales and well coupled to the gas, so that the magnetic stress tensor reduces to an isotropic pressure that is polytropic, with index $\gamma_{\mathrm{m}}=4 / 3$ (Henney et al. 2005). The magnetic field of the entire cloud is then set by the field at the illuminated face, increasing as $B \sim \rho^{2 / 3}$. The magnetic field in Orion-S is not known but fields are important elsewhere in the Orion environment (Abel et al. 2004a). The initial magnetic field was taken as $3 \mu \mathrm{G}$, which rises to $850 \mu \mathrm{G}$ deep inside the cloud, where the combined magnetic and turbulent pressures become roughly equal to the thermal pressure. We found that models with stronger magnetic fields were not capable of reproducing 

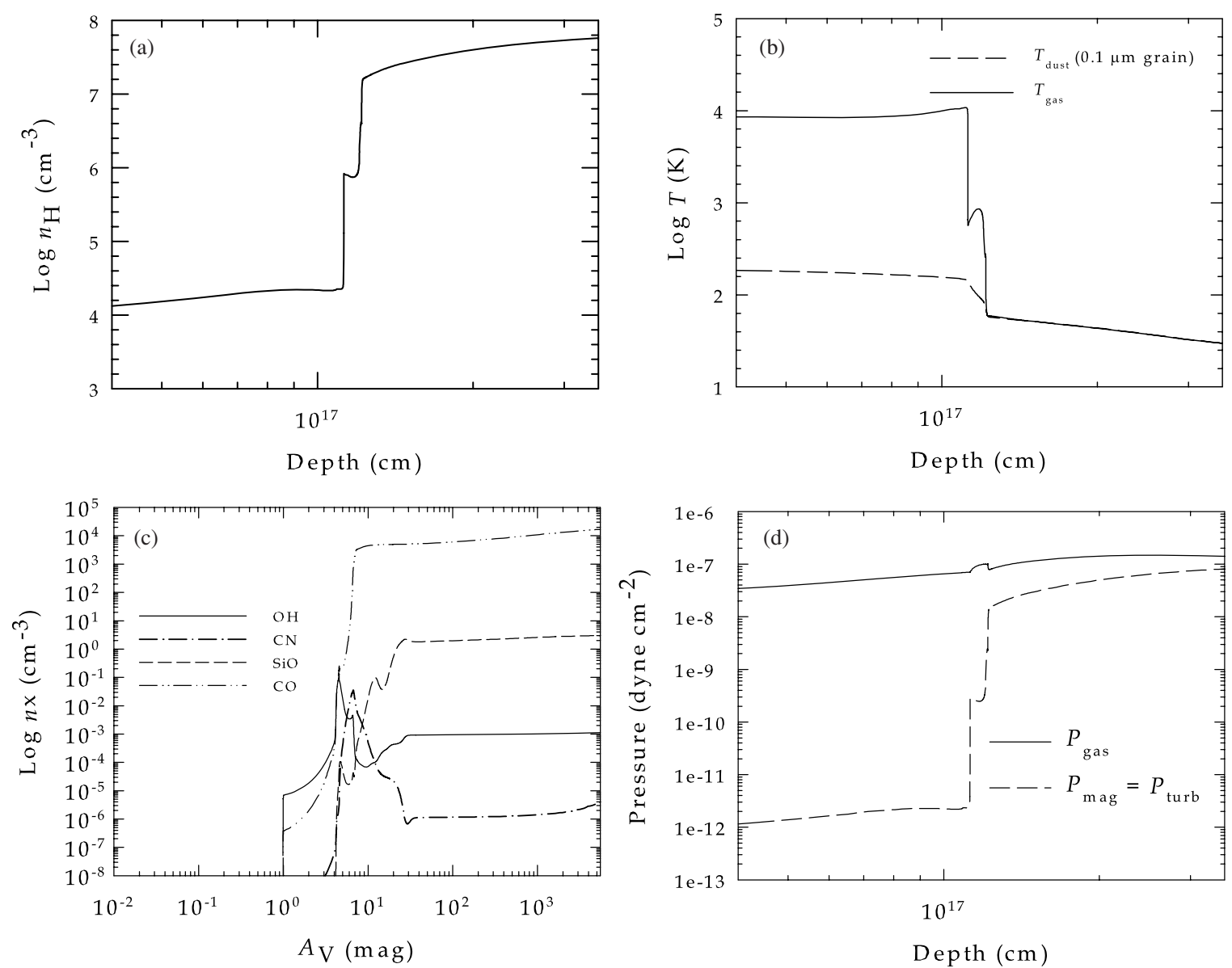

Figure 7. Physical structure for our constant-pressure model of the ionized, atomic, and molecular gas in the Orion-S Cloud. Plotted as a function of depth into the model are the (a) density, (b) gas and dust temperatures (c), molecular abundances, and (d) pressure constituents.

the observed molecular hydrogen density deep in the cloud of $n \simeq 3 \times 10^{7} \mathrm{~cm}^{-3}$, since factors 5 and 6 of Section 3.3 .1 were too effective in counteracting factor 3 .

We assume a closed geometry, in which all radiation must eventually escape in the direction away from the central star cluster, which is appropriate if optically thick gas fully surrounds the cluster. Under this assumption, inwardly directed diffuse emission is compensated by symmetric emission from the other side of the star cluster. Light can undergo multiple scatterings as it diffuses outward though the cloud, so that the total rate of momentum transfer exceeds the single scattering limit, $L_{*} / c$, by a few factors. We use the McMullun et al. (1993) deduced hydrogen column density to set the total thickness of the cloud.

The entire structure and properties of the cloud follows from these assumptions. Cloudy computes the kinetic temperature, ionization, chemistry, optical depths, and emission properties of the gas at each point. The density and radiation field striking the illuminated face are set by observations and the gas pressure at this point is computed. The calculation follows the starlight as it penetrates the cloud. The transmitted continuum sets the pressure and physical conditions. The magnetic field follows from our flux-freezing assumption. The total pressure is set by the assumption of magnetostatic equilibrium. The calculation stops when the observed column density is reached.

Figure 7 shows the results of our calculations for the density, temperature, molecular abundances, and pressure as a function of depth into the cloud. The first criterion we demand our model reproduce is clearly satisfied: that the model yields a temperature high enough to allow $\mathrm{H}_{2} \mathrm{CO}$ to evaporate off of grain surfaces and remain in the gas phase. The dust temperature (Figure 7(b)) remains above $38 \mathrm{~K}$ throughout the model, which, as discussed above in Section 3.3.1, is high enough to inhibit freeze-out of $\mathrm{H}_{2} \mathrm{CO}$. This is achieved in our model purely through external heating by starlight from the Trapezium.

Although we can explain the presence of gas-phase $\mathrm{H}_{2} \mathrm{CO}$ using our model, the chemistry of Orion-S is extremely complex. At our MC temperature of $38 \mathrm{~K}$, some molecules, such as $\mathrm{SiO}$, $\mathrm{CS}$, and $\mathrm{SO}_{2}$, will freeze out onto grains. Figure 7(c) shows the predicted $\mathrm{CO}, \mathrm{CN}, \mathrm{OH}$, and $\mathrm{SiO}$ abundance as a function of $A_{V}$. Our prediction of $\mathrm{SiO}$, once the full effects of freeze out and molecular evaporation off grain surfaces are included, will likely be quite different. The current version of Cloudy only treats freeze-out and evaporation processes involving $\mathrm{CO}$, $\mathrm{OH}$, and $\mathrm{H}_{2} \mathrm{O}$, with the goal of making accurate predictions of gas-phase oxygen deep in MCs. The freeze-out of other atoms/ molecules, such as $\mathrm{SiO}$, is not considered by our models, and neither are grain-surface reactions, other than those that form $\mathrm{H}_{2}$. So, while our current analysis is sufficient to deduce that $\mathrm{H}_{2} \mathrm{CO}$ remains in the gas phase, a full treatment of gas-grain and timedependent chemical effects is necessary to fully understand all the rich chemical environment of Orion-S. ${ }^{12} \mathrm{CO}$ and ${ }^{13} \mathrm{CO}$ lines are optically thick and not sensitive to gas properties such as pressure or column density. $\mathrm{C}^{17} \mathrm{O}$ and $\mathrm{C}^{18} \mathrm{O}$ should be optically thin and therefore potentially a good diagnostic of physical properties. However, as Orion-S and OMC1 lie along the same sightline with similar kinematic properties, the optically thin CO 
emission from $\mathrm{OMC} 1$ and Orion-S is difficult to disentangle. Therefore, we do not present $\mathrm{CO}$ comparisons because of these complexities.

While our current analysis is sufficient to deduce $\mathrm{H}_{2} \mathrm{CO}$ remains in the gas phase, a full treatment of gas-grain and time-dependent chemical effects is necessary to fully understand the chemical environment of Orion-S. Such a treatment, while beyond the scope of this work and the current version of Cloudy (Cloudy currently only treats freeze-out of $\mathrm{CO}, \mathrm{OH}$, and $\mathrm{H}_{2} \mathrm{O}$ ), will be the subject of a future investigation.

One appealing aspect of our model is how we succeed in reproducing the projected dimensions of Orion-S under the assumption of magnetostatic equilibrium, with an effective gravity provided by the momentum of the absorbed starlight. The density (Figure $7(\mathrm{a})$ ) varies by $\sim 4$ dex over the physical extent of our model, yet our calculation reaches $N(\mathrm{H}) \sim$ $4 \times 10^{24} \mathrm{~cm}^{-2}$ at a depth of $2.4 \times 10^{17} \mathrm{~cm}$, or within $20 \%$ of the observed projected thickness. Despite this success, there are arguably some deficiencies in our model assumptions, of which the most serious are the neglect of self-gravity and the rather weak magnetic field. Using the observed mass and size of the cloud (Section 3.3.1), one finds the escape speed at the cloud surface to be $7.3 \mathrm{~km} \mathrm{~s}^{-1}$, meaning that the molecular gas is gravitationally bound, while the ionized gas is gravitationally unbound. Thus, factor 3 of Section 3.3.1 is likely to contribute significantly to the overpressure of the molecular gas, so that a self-gravitating model would allow the magnetic field in the cloud to be significantly higher, while still satisfying the observational constraints. However, it is not clear that any equilibrium self-gravitating model is possible. Unless the turbulent and magnetic pressures are more than 100 times greater than given by our model, the cloud mass is higher than both the Jeans critical value and the magnetic critical value, and so the cloud may be dynamically collapsing on a free-fall timescale of a few times $10^{4}$ years.

In summary, our equilibrium Cloudy model represents a necessary compromise, including as it does a detailed simulation of the relevant microphysics, but at the expense of a simplified treatment of some of the relevant macrophysics. As such, although it is broadly successful in accounting for the observed properties of the Orion-S Cloud using almost no free parameters, it should not be considered the last word in modeling this region, especially in the light of the uncertainties outlined in the previous paragraph.

\subsection{What is the Impact of a Putative High Relative Velocity of $\theta^{1}$ Ori C?}

In Section 2.7, we saw that $\theta^{1}$ Ori $\mathrm{C}$ appears to be moving away from the $\mathrm{OMC}$ at about $13 \mathrm{~km} \mathrm{~s}^{-1}$. Since this star is only about $0.2 \mathrm{pc}$ in front of the local MIF, $\theta^{1}$ Ori $\mathrm{C}$ would have been at the present location of the MIF only 15,000 years ago. This is simply a reference number for the dynamic timescale as one would expect that $\theta^{1}$ Ori $\mathrm{C}$ would have continuously ionized the gas immediately surrounding it, thus producing a constantly changing structure. It does appear that $\theta^{1}$ Ori C has only recently entered a region of low-density gas and has just begun to photoionize a much larger volume of space (the Huygens region and the EON). This is a dramatically new way of viewing the Orion Nebula and means that the object need not be in quasi-dynamic equilibrium. A short illumination age of the ONC stars is quite attractive because the well-determined mass-loss rates of the proplyds close to $\theta^{1}$ Ori $\mathrm{C}$ indicate that they should have been destroyed through photoablation within the last $1.5 \times 10^{4}$ to $1.1 \times 10^{6}$ years (Churchwell et al. 1987 ; Henney et al. 2002; Smith et al. 2005; O’Dell \& Henney 2008). The absence of any evidence for destruction indicates that their illumination ages by $\theta^{1}$ Ori $\mathrm{C}$ are no more than a few times 1.5 $\times 10^{4}$ years.

If one accepts the scenario that $\theta^{1}$ Ori $\mathrm{C}$ has emerged from a much higher density environment during the last 10,000 years, then it is worth considering how this picture would change the expected ionization, density, and dynamics of the present-day nebula. If the surrounding density was much higher in the past, then the $\mathrm{H}$ II region would have been much smaller. For example, in an ambient density of $n=10^{6} \mathrm{~cm}^{-3}$, the Strömgren radius would be only 0.007 , or $3^{\prime \prime} 2$. As $\theta^{1}$ Ori C left the high-density environment, an R-type ionization front would propagate into the low-density gas at a speed of $\Phi_{\mathrm{H}} / n \geqslant 1000 \mathrm{~km} \mathrm{~s}^{-1}$, where $\Phi_{\mathrm{H}}$ is the ionizing flux, defined in Section 2.6.2. This speed is much larger than the stellar velocity, so that deviations from static photoionization equilibrium would be fleeting and difficult to detect. Assuming a density $n=100 \mathrm{~cm}^{-3}$ for the lowdensity gas, the ionization front would reach its equilibrium Strömgren value $(\simeq 3 \mathrm{pc})$ in only 1000 years. The maximum recombination front velocity is also of order $1000 \mathrm{~km} \mathrm{~s}^{-1}$ so that the gas would recombine effectively instantaneously in the high-density regions vacated by the star. The timescale for molecular hydrogen formation on dust grains is approximately $1000\left(10^{6} \mathrm{~cm}^{-3} / n\right)$ yr (Tielens \& Hollenbach 1985 ), so that, unless the previously ionized gas was of rather low density, there has been time for it to return to a fully molecular state.

The density structure of the ionized gas is intimately tied to its dynamics, with typical internal velocities of order the ionized sound speed, which is very similar to the putative stellar velocity of $13 \mathrm{~km} \mathrm{~s}^{-1}$. It seems likely, therefore, that the ionized density structure will not be greatly affected by the stellar motion. Numerical simulations confirm (Henney et al. 2005; Arthur \& Hoare 2006) that an approximately steady-state champagne flow is established on a timescale equal to the sound crossing time. It is therefore at the largest scales in the nebula $(>1)$ that one would expect to find the clearest evidence for nonequilibrium dynamics induced by the stellar motion. However, uncertainties in the exact ambient density distribution and the effect of the SW would make it difficult to draw clear conclusions. Another possible observational diagnostic of a moving ionized star would be the dense neutral wake, which numerical simulations predict should form behind the star (Raga et al. 1997; Kraus et al. 2007). Again, observational detection of such a wake would be very challenging, chiefly due to confusion brought on by the rich profusion of structures at all scales in the MC behind the nebula.

A very young age for the present configuration of the Orion Nebula could also remove the dilemma presented by the fact that the $\mathrm{H}^{+}$layer lying between $\theta^{1}$ Ori $\mathrm{C}$ and the Veil is moving toward the Veil with a closure velocity of about $18 \mathrm{~km} \mathrm{~s}^{-1}$ and there is no evidence of an interaction with the Veil. Abel et al. (2006) calculate that the collision time with the Veil is about 40,000 years. Without a detailed model of the expected very time-dependent structure, one cannot assess the importance of this number except that it too indicates that the present configuration is quite young.

In conclusion, it seems impossible to definitively confirm or rule out the moving star hypothesis, based on existing observational evidence. The strongest argument in favor remains the short mass-loss lifetimes derived for the proplyds in the inner nebula. The strongest arguments against are (1) the required 
coincidence that $\theta^{1}$ Ori $\mathrm{C}$ currently resides so close to the center of the Trapezium cluster, despite not being dynamically bound to the cluster and (2) the difficulty in explaining how $\theta^{1}$ Ori $\mathrm{C}$ acquired such a high velocity. The mass of the current $\theta^{1}$ Ori $\mathrm{C}$ multiple system is $\simeq 50 M_{\odot}$ (Kraus et al. 2007), so assuming that it originally had a velocity typical of ONC members, then to achieve its current velocity it must have ejected a $\simeq 10 M_{\odot}$ companion at $65 \mathrm{~km} \mathrm{~s}^{-1}$, or even faster for a less-massive star. Note that these high stellar velocities mean that many-body interactions at the level of the star cluster are unlikely to play a role since the velocity dispersion of the cluster is only a few $\mathrm{km} \mathrm{s}^{-1}$ (Jones \& Walker 1988) and disruption of binaries by the cluster potential has been shown to be ineffective for binary separations < 0.'5 (Reipurth et al. 2007).

One further possibility examined is that the velocity kick of the ionizing star was acquired much more recently. This would sidestep most of the arguments discussed above, both for and against the moving star hypothesis. For example, Tan $(2004,2008)$ has proposed that the runaway B star known as the BN object (Becklin \& Neugebauer 1967) was ejected from the $\theta^{1}$ Ori $\mathrm{C}$ multiple system 4500 years ago (but see Gómez et al. 2005, 2008 for an alternative viewpoint). If the anomalous velocity of $\theta^{1}$ Ori $\mathrm{C}$ is due to this event, then it will only have moved about $0.05 \mathrm{pc}$ since then, which is very small compared with the size of the nebula, so that observable consequences are unlikely. However, one serious problem with this proposal is that the measured radial velocity of BN is $+13 \mathrm{~km} \mathrm{~s}^{-1}$ with respect to the ONC rest frame (Scoville et al. 1983), which is the same absolute value as that recently determined for $\theta^{1}$ Ori C (Stahl et al. 2008), but in the opposite direction. BN's midIR luminosity of $2600 L_{\odot}$ (Gezari et al. 1998; re-adjusted to a distance of $440 \mathrm{pc}$ ) is consistent with a dust-enshrouded mainsequence B2 to B3 star, implying a mass of $9 \pm 1 M_{\odot}$, which is more than five times less than the mass of $\theta^{1}$ Ori $\mathrm{C}$. Therefore, as discussed in the previous paragraph, momentum conservation means that $\mathrm{BN}$ ought to have a much higher redshifted velocity than is observed if it has truly been ejected from the $\theta^{1}$ Ori $\mathrm{C}$ system in the recent past.

\subsection{Summary and Conclusions}

In this paper, we have been able to establish several remarkable new properties of the Orion Nebula. (1) There is an incomplete shell of high-ionization gas shaped by the high-velocity wind arising from $\theta^{1}$ Ori $C$ and open in the direction of detected X-ray emission. (2) The highest surface brightness portion of the Orion Nebula occurs to the southwest of the Trapezium stars, in the region called Orion-S, a center of star formation and stellar outflow. (3) The Orion-S region is caused by an optically thick $\mathrm{MC}$ which lies within the ionized cavity of the Orion Nebula. This Orion-S Cloud is the host of a separate region of star formation and is sufficiently cold in the middle that it can produce $\mathrm{H}_{2} \mathrm{CO}$ lines in absorption. (4) It is possible that $\theta^{1}$ Ori C has a large radial velocity with respect to the $\mathrm{ONC}$ and other members of the Trapezium. The observations of the nebula do not contradict such a possibility, and the presence of a large radial velocity would resolve the conundrum of the lack of destruction of the disks in proplyds close to $\theta^{1}$ Ori $\mathrm{C}$ and would explain why the inner $\mathrm{H}^{+}$layer expanding toward the observer has not yet reached the Veil feature.

C.R.O.'s work was supported in part by the Space Telescope Science Institute grants GO 10921 and GO 10967. N.P.A. acknowledges financial support through the National Science
Foundation (NSF) under Grant No. 0094050, 0607497 to the University of Cincinnati. G.J.F. thanks the NSF (AST 0607028) and NASA (NNG05GD81G) for support. W.J.H. and S.J.A. acknowledge financial support from DGAPA-UNAM, Mexico (PAPIIT IN110108 and IN100309).

\section{Facilities: HST (WFPC2)}

\section{REFERENCES}

Abel, N. P, Brogan, C. L, Ferland, G. J., O’Dell, C. R., Shaw, G., \& Troland, T. H. 2004a, ApJ, 609, 247

Abel, N. P., Ferland, G. J., O'Dell, C. R., Shaw, G., \& Troland, T. H. 2004b, ApJ, 609, 247

Abel, N. P., Ferland, G. J., O’Dell, C. R., \& Troland, T. H. 2006, ApJ, 644, 344 Abel, N. P., Ferland, G. J., Shaw, G., \& van Hoof, P. A. M. 2005, ApJS, 161, 65 Abel, N. P., van Hoof, P. A. M., Ferland, G. J., Shaw, G., \& Elwert, T. 2008, ApJ, 686, 1125

Abt, H. A., Wang, R., \& Cardona, O. 1991, ApJ, 367, 155

Allen, C., Poveda, A., \& Worley, C. E. 1974, RevMexAA, 1, 101

Arthur, S. J. 2007, in Diffuse Matter from Star Forming Regions to Active Galaxies, ed. T. W. Hartquist, J. M. Pittard, \& S. A. E., G. Falle (Dordrecht: Springer) 183

Arthur, S. J., \& Hoare, M. G. 2006, ApJS, 165, 283

Baldwin, J. A., Ferland, G. J., Martin, P. G., Corbin, M. R., Cota, S. A., Peterson, B. M., \& Slettebak, A. 1991, ApJ, 374, 580

Ballick, B., Gammon, R. H., \& Hjellming, R. M. 1974, PASP, 86, 616

Bally, J., O’Dell, C. R., \& McCaughrean, M. J. 2000, AJ, 119, 2919

Bally, J., Sutherland, R. S., Devine, D., \& Johnstone, D. 1998, AJ, 116, 293

Becklin, E. E., \& Neugebauer, G. 1967, ApJ, 147, 799

Bertoldi, F. 1989, ApJ, 346, 735

Blaauw, A., \& Morgan, W. W. 1954, ApJ, 119, 625

Cantó, J., Raga, A., Steffen, W., \& Shapiro, P. R. 1998, ApJ, 502, 695

Cardelli, J. A., Clayton, G. C., \& Mathis, J. S. 1989, ApJ, 345, 245

Churchwell, E., Felli, M., Wood, D. O. S., \& Massi, M. 1987, ApJ, 321, 516

Costero, R., \& Peimbert, M. 1970, Bol. Obs. Tonantzintla Tacubaya, 5, 229

Crutcher, R. M. 1999, ApJ, 520, 706

Doi, T., O’Dell, C. R., \& Hartigan, P. 2002, AJ, 124, 445

Doi, T., O'Dell, C. R., \& Hartigan, P. 2004, AJ, 127, 3456

Donati, J.-F., Babel, J., Harries, T. J., Howarth, I. D., Petit, P., \& Semel, M. 2002, MNRAS, 333, 55

Dyson, J. E. 1968, Ap\&SS, 1, 388

Dyson, J. E., \& de Vries, J. 1972, A\&A, 20, 223

Dyson, J. E., \& Williams, D. A. 1997, The Physics of the Interstellar Medium, Graduate Series in Astronomy (2nd ed.; Bristol: Institute of Physics Publishing)

Esteban, C., Peimbert, M., Torres-Peimbert, S., \& Escalante, V. 1998, MNRAS, 295, 401

Felli, M., Churchwell, E., Wilson, T. L., \& Taylor, G. B. 1993, A\&AS, 98, 137

Ferland, G. J., Korista, K. T., Verner, D. A., Ferguson, J. W., Kingdon, J. B., \& Verner, E. M. 1998, PASP, 110, 76

Fürész, G., Hartmann, L. W., Megeather, S. T., Sventgyorgyi, A. H., \& Hamden, E. T. 2008, ApJ, 676, 1109

Gagné, M., Oksala, M. E., Cohen, D. H., Tonnesen, S. K., ud-Doula, A., Owocki, S. P., Townsend, R. H. D., \& MacFarlane, J. J. 2005, ApJ, 628, 986

García-Arredondo, F., Arthur, S. J., \& Henney, W. J. 2002, RevMexAA, 38, 51

García-Arredondo, F., Henney, W. J., \& Arthur, S. J. 2001, ApJ, 561, 830

García-Díaz, Ma.-T., \& Henney, W. J. 2007, AJ, 133, 952

García-Díaz, Ma.-T., Henney, W. J., López, J. A., \& Doi, T. 2008, RevMexAA, 44,181

García-Segura, G., \& Franco, J. 1996, ApJ, 469, 171

Gaume, R. A., Wilson, T. L., Vrba, F. J., Johnston, K. J., \& Schmid-Burgk, J. 1998, ApJ, 493, 940

Gezari, D. Y., Backman, D. E., \& Werner, M. W. 1998, ApJ, 509, 283

Gómez, L., Rodríguez, L. F., Loinard, L., Lizano, S., Allen, C., Poveda, A., \& Menten, K. M. 2008, ApJ, 685, 333

Gómez, L., Rodríguez, L. F., Loinard, L., Lizano, S., Poveda, A., \& Allen, C. 2005, ApJ, 635, 1166

Güdel, M., Briggs, K. R., Montmerle, T., Audard, M., Rebull, L., \& Skinner, S. L. 2008, Science, 319, 309

Hartquist, T. W., Dyson, J. E., Pettini, M., \& Smith, L. J. 1986, MNRAS, 221 , 715

Hasegawa, T., \& Herbst, E. 1993, MNRAS, 263, 589

Hasegawa, T., Herbst, E., \& Leung, C. M. 1992, ApJS, 82, 167

Hayward, T. L., Houck, J. R., \& Miles, J. W. 1994, ApJ, 433, 157

Heiles, C., Chu, Y.-H., \& Troland, T. H. 1981, ApJ, 247, L77 
Henney, W. J., Arthur, S. J., \& García-Díaz, Ma.-T. 2005a, ApJ, 627, 813

Henney, W. J., \& O'Dell, C. R. 1999, AJ, 118, 2350

Henney, W. J., O’Dell, C. R., Meaburn, J., Garrington, S. J., \& López, J. A. 2002, ApJ, 566, 315

Henney, W. J., O’Dell, C. R., Zapata, L. A., García-Díaz, Ma. T., Rodríguez, L. F., \& Robberto, M. 2007, AJ, 133, 2343

Hillenbrand, L. A., \& Carpenter, J. M. 2000, ApJ, 540, 236

Hoogerwerf, R., de Bruijne, J. H. J., \& de Zeeuw, P. T. 2001, A\&A, 365, 49

Houde, M., Dowell, C. D., Hildebrand, R. H., Dotson, J. L., Vaillancourt, J. E., Phillips, T. G., Peng, R., \& Bastien, P. 2004, ApJ, 604, 717

Ikeda, N., Sunada, K., \& Kitamura, Y. 2007, ApJ, 665, 1194

Johnston, K. J., Palmer, P., Wilson, T. L., \& Bieging, J. H. 1983, ApJ, 271, L89

Jones, B. F., \& Walker, M. F. 1988, AJ, 95, 1755

Kaify, N. T., et al. 2000, PASJ, 52, 1

Kraus, S. Y. Y., et al. 2007, A\&A, 466, 649

Mangum, J. G., Wootten, Al, \& Plambeck, R. L. 1993, ApJ, 409, 282

McMullun, J. P., Mundy, L. G., \& Blake, G. A. 1993, ApJ, 405, 599

Megeath, T., \& Robberto, M. 2006, Spitzer Space Telescope (Pasadena, CA: Cal Tech), ssc2006-21a

Mellema, G., Arthur, S. J., Henney, W. J., Iliev, I. T., \& Shapiro, P. R. 2006, ApJ, 647, 397

Mesa-Delgado, A., Esteban, C., \& García-Rojas, J. 2008, ApJ, 675, 389

Muench, A., German, K., Hillenbrand, L., \& Preibisch, T. 2008, in Handbook of Star Forming Regions, Vol. 4, ed. B. Reipurth (San Francisco, CA: ASP), 483

O'Dell, C. R. 2000, AJ, 119, 2311

O'Dell, C. R. 2001a, ARAA, 39, 99

O'Dell, C. R. 2001b, PASP, 113, 29

O'Dell, C. R. 2002, RevMexAA, 12, 12

O'Dell, C. R. 2004, PASP, 116, 729

O'Dell, C. R., \& Doi, T. 1999, PASP, 111, 1316

O’Dell, C. R., Hartigan, P., Bally, J., \& Morse, J. A. 1997, AJ, 114, 2016

O'Dell, C. R., \& Henney, W. J. 2008, AJ, 136, 1566

O’Dell, C. R., Muench, A., Smith, N., \& Zapata, L. 2008, in Handbook of Star Forming Regions, Vol. 4, ed. B. Reipurth (San Francisco, CA: ASP), 544

O'Dell, C. R., Peimbert, M., \& Peimbert, A. 2003, AJ, 125, 2590

O’Dell, C. R., Walter, D. K., \& Dufour, R. J. 1992, ApJ, 399, L67

O’Dell, C. R., \& Wen, Z. 1994, ApJ, 436, 194

O'Dell, C. R., \& Wong, S.-K. 1996, AJ, 111, 846

O'Dell, C. R. \& Yusef-Zadeh, F. 2000, AJ, 120, 382

Osterbrock, D. E., \& Ferland, G. J. 2006, Astrophysics of Gaseous Nebulae and Active Galactic Nuclei (2nd ed.; Sausalito, CA: Univ. Science Books)

Patience, J., Zavala, R. T., Prato, L., Franz, O., Wasserman, L., Tycner, C., Hutter, D. J., \& Hummel, C. A. 2008, ApJ, 674, 97

Pellegrini, E. W. J. A., et al. 2007, ApJ, 658, 1119
Pflamm-Altenburg, J., \& Kroupa, P. 2006, MNRAS, 373, 295

Pikel'ner, S. B. 1968, Astrophys. Lett., 2, 97

Pogge, R. W., Owen, J. M., \& Atwood, B. 1992, ApJ, 399, 147

Raga, A. C., Noriega-Crespo, A., Cantó, J., Steffen, W., van Buren, D., Mellema, G., \& Lundqvist, P. 1997, RevMexAA, 33, 73

Reipurth, B., Guimarães, M. M., Connelley, M. S., \& Bally, J. 2007, AJ, 134 2272

Robberto,, et al. 2005, AJ, 129, 1534

Rodríguez, L. F., Poveda, A., Lizano, S., \& Allen, C. 2005, ApJ, 627, L65

Scoville, N., Kleinmann, S. G., Hall, D. N. B., \& Ridgway, S. T. 1983, ApJ, 275, 201

Smith, N., Bally, J., Shuping, R. Y., Morris, M., \& Hayward, T. L. 2004, ApJ, 610, L117

Smith, N., Bally, J., Shuping, R. Y., Morris, M., \& Kassis, M. 2005, AJ, 130 1763

Smith, M. A., \& Fullerton, A. W. 2005, PASP, 117, 13

Stahl, O., Wade, G., Petit, V., Stober, B., \& Schanne, L. 2008, A\&A, 487, 323

Stahl, O., Wolf, B., Gang, T., Gummersbach, C. A., Kaufer, A., Kovacs, J., Mandel, H., \& Szeifert, T. 1993, A\&A, 274, L29

Stahl, O. A., et al. 1996, A\&A, 312, 539

Stanke, T., McCaughrean, M. J., \& Zinneker, H. 2002, A\&A, 392, 239

Stone, R. C. 1991, AJ, 101, 333

Subrahmanyan, R., Goss, W. M., \& Malin, D. F. 2001, AJ, 121, 399

Tan, J. C. 2004, ApJ, 607, L47

Tan, J. C. 2008, arXiv:0807.3771

Tielens, A. G. G. M., \& Hollenbach, D. 1985, ApJ, 291, 722

van Altena, W. F., Lee, J. T., Lee, J.-F., Lu, P. K., \& Upgren, A. R. 1988, AJ, 95,1744

van der Werf, P. P., \& Goss, J. M. 1989, ApJ, 224, 409

Vázquez-Semadeni, E., Kim, J., Shadmehri, M., \& Ballesteros-Paredes, J. 2005, ApJ, 618, 344

Vitrichenko, E. A. 2002, Astron. Lett., 28, 324

Wade, G. A., Fullerton, A. W., Donati, J.-F., Landstreet, J. D., Petit, P., \& Strasser, S. 2006, A\&A, 451, 195

Wen, Z., \& O'Dell, C. R. 1995, ApJ, 438, 784

Wilson, T. L., Muders, D., Kramer, C., \& Henkel, C. 2001, ApJ, 557, 240

Zapata, L. A., Ho, P. T. P., Rodríguez, L. F., O’Dell, C. R., Zhang, Q., \& Muench, A. 2006, ApJ, 653, 398

Zapata, L. A., Rodríguez, L. F., Ho, P. T. P., Zhang, Q., Chunhua, Q., \& Kurtz, S. E. 2005, ApJ, 630, L85

Zapata, L. A., Rodríguez, L. F., Kurtz, S. E., \& O'Dell, C. R. 2004a, AJ, 127, 2252

Zapata, L. A., Rodríguez, L. F., Kurtz, S. E., O’Dell, C. R., \& Ho, P. T. P. 2004b, ApJ, 610, L121

Zuckerman, B. 1973, ApJ, 183, 863 